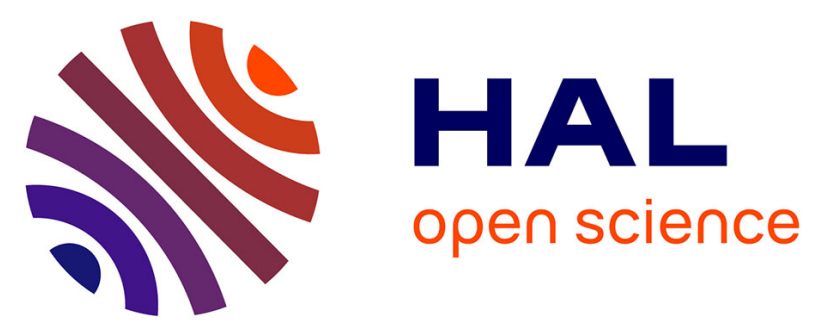

\title{
Identité technique et comportements économiques des groupes proto-aurignaciens à la grotte de l'Observatoire (principauté de Monaco)
}

\author{
Guillaume Porraz, Patrick Simon, Amaranta Pasquini
}

\section{- To cite this version:}

Guillaume Porraz, Patrick Simon, Amaranta Pasquini. Identité technique et comportements économiques des groupes proto-aurignaciens à la grotte de l'Observatoire (principauté de Monaco). Gallia Préhistoire - Préhistoire de la France dans son contexte européen, 2010, 52, pp.33-59. 10.3406/galip.2010.2470 . halshs-00586193

\section{HAL Id: halshs-00586193 \\ https://shs.hal.science/halshs-00586193}

Submitted on 3 Jan 2020

HAL is a multi-disciplinary open access archive for the deposit and dissemination of scientific research documents, whether they are published or not. The documents may come from teaching and research institutions in France or abroad, or from public or private research centers.
L'archive ouverte pluridisciplinaire HAL, est destinée au dépôt et à la diffusion de documents scientifiques de niveau recherche, publiés ou non, émanant des établissements d'enseignement et de recherche français ou étrangers, des laboratoires publics ou privés.

\section{(ㅇ)(1) $\$$}

Distributed under a Creative Commons Attribution - NonCommercial - NoDerivatives $\mid 4.0$ 


\title{
IDENTITÉ TECHNIQUE ET COMPORTEMENTS ECONOMIQUES DES GROUPES PROTO-AURIGNACIENS À LA GROTTE DE L'OBSERVATOIRE (PRINCIPAUTÉ DE MONACO)
}

\author{
Guillaume PORRAZ*, Patrick SIMON** et Amaranta PASQUINI***
}

\begin{abstract}
Mots-clés. Arc méditerranéen, approvisionnement minéral, production lithique, schéma de mobilité, diffusion, hommes anatomiquement modernes.

Résumé. Cet article présente de premiers résultats et réflexions sur l'assemblage lithique proto-aurignacien de la grotte de l'Observatoire (principauté de Monaco) et fait valoir l'intérêt de ce contexte régional pour l'étude de la diffusion du Paléolithique supérieur en Europe occidentale.

Cette étude confirme l'attribution des niveaux $G$ et F au complexe proto-aurignacien (Onoratini et al., 1999). Les chaînes opératoires lithiques se caractérisent par une acquisition sélective de roches de très bonne qualité, par la coexistence de deux schémas de production unipolaire, l'un laminaire, l'autre lamellaire, et par une confection de lamelles à dos par retouche inverse dextre (lamelle Dufour, soustype Dufour). Ces caractéristiques sont proches de celles aujourd'hui décrites dans la littérature, laissant ainsi entrevoir l'homogénéité technique de ce complexe à l'échelle de l'Europe occidentale.

Le profil de cet assemblage caractérise des occupations de courte durée, renvoyant à des stratégies de subsistance basées sur une forte mobilité résidentielle et de longs déplacements (> $130 \mathrm{~km}$ ). Dans cette perspective, les données techno-économiques se prêtent à l'hypothèse d'un complexe technoculturel porté par des nouvelles populations qui auraient circulé le long de l'espace méditerranéen. La localisation principale des gîtes d'approvisionnement en matières premières ( $85 \%$ de silex bédouliens et oligocènes de Provence occidentale) nuancerait alors l'hypothèse d'une avancée continue des premiers hommes anatomiquement modernes en Europe, privilégiant plutôt le scénario d'une progression ponctuée de stases.
\end{abstract}

Key-words. Mediterranean Arc, Proto-aurignacian, raw material provisioning, lithic technology, settlement patterns, diffusion, anatomically modern humans.

Abstract. This paper introduces first results and comments concerning the Proto-Aurignacian lithic assemblage from the grotte de l'Observatoire (Principality of Monaco) and highlights the importance of this area for our perception of the earlier phase of the Upper Palaeolithic in Western Europe.

This study confirms the attribution of layers $G$ and $F$ to the Proto-Aurignacian complex (Onoratini et al., 1999). The lithic reduction sequences are characterized by a selection of raw materials of high suitability, the coexistence of two unipolar production systems, one laminar, one lamellar, and by a typological corpus dominated by lateralized and inversely retouched bladelets (Dufour bladelet, subtype Dufour). These technological signatures are similar to those described in the current literature, giving the impression of a homogeneous techno-complex across Western Europe.

The features of the lithic assemblage characterize short-term occupations, within a framework of a high residential mobility and long distances of circulation $(>130 \mathrm{~km})$. In that sense, the techno-economical data fit with the scenario of the arrival of the first anatomically

\footnotetext{
* Université de Tübingen, Schloss, Burgsteige 11, 72070 Tübingen, Allemagne. Courriel: guillaume.porraz@ifu.uni-tuebingen.de

** Musée d'Anthropologie préhistorique de Monaco, 56bis boulevard du Jardin-Exotique, MC-98000 Monaco. Courriel: patrick.simon@map-mc.com *** Lampea, Maison méditerranéenne des sciences de l'homme, Université de Provence, 5 rue du Château-de-l'Horloge, BP 647, F-13094 Aix-enProvence Cedex 2 et Universitat Autònoma de Barcelona (UAB), España. Courriel: amerasi@gmail.com
} 
modern humans who would have followed the Mediterranean Arc as a way of dispersion. However, the location of the lithic raw materials sources ( $85 \%$ of the raw materials come from the west) nuances the hypothesis of a continuous and gradual progression of these pioneering populations. Data suggests a scenario of a human dispersion characterized by episodes of stasis.

Les synthèses et travaux les plus récents reconnaissent aujourd'hui l'antériorité du Proto-Aurignacien sur l'Aurignacien ancien (voir Laplace, 1966; Bon, 2002 et 2005; Bordes et al., 2008; Bar-Yosef, 2006; Teyssandier, 2007), modifiant ainsi substantiellement les schémas historiques de ce début du Paléolithique supérieur en Europe occidentale. Quoique non démontrée jusqu'alors, l'hypothèse implicite est celle d'un complexe intrusif d'origine orientale lié à la première vague de peuplement des hommes anatomiquement modernes sur ce continent (Bar-Yosef, 2003; Mellars, 2006; Zilhao, 2006). Les datations obtenues à ce jour identifient un complexe proto-aurignacien de durée relativement brève, présent au cours du trente-huitième millénaire BP (cal. 38-36000 BP) et pour partie contemporain des industries dites de transition. Cet ensemble technoculturel, initialement reconnu dans le Bassin méditerranéen, s'étendrait géographiquement de l'Europe orientale jusqu'à ses rivages atlantiques (Teyssandier, 2007).

Ce processus de dispersion des populations d'hommes anatomiquement modernes en Europe se double d'un processus de substitution aux populations néandertaliennes locales. Cette trame historique implique différentes formes d'interactions (de l'absence à la «fusion»), différentes étapes dans le processus de remplacement et enfin différentes chronologies selon les régions considérées et observées. Consécutivement, elle implique l'existence de signatures archéologiques diverses, pouvant souligner différents états au sein d'un même processus. L'étude de l'apparition du Paléolithique supérieur en Europe revient donc à décliner des scénarios régionaux aux géographies physiques, économiques et humaines contrastées. Les stratégies d'observation sont importantes, les réflexions épistémologiques non moins fondatrices (Guillomet-Malmassari, 2009).

La reprise pour étude de l'industrie lithique protoaurignacienne de la grotte de l'Observatoire à Monaco s'inscrit initialement dans cette volonté de découper les espaces étudiés afin d'en mesurer les implications théoriques. La grotte de l'Observatoire se trouve au sein de l'aire liguro-provençale, entité géomorphologique qui s'étend sur près de $400 \mathrm{~km}$ de long, limitée au nord par les chaînons subalpins et au sud par la mer Méditerranée. Cette bande littorale, qui permet de relier la plaine du Pô au sillon rhodanien, constitue un axe de circulation humaine privilégié. Pour l'étude du Proto-Aurignacien, les données et travaux de synthèse favorisent communément l'hypothèse d'une diffusion initiale le long de cet axe méditerranéen (voir Kozlowski, Otte, 2000; Mellars, 2004; Bar-Yosef, 2006).

Les résultats et premières réflexions de cette étude reposent sur une caractérisation du sous-système technique lithique, impliquant une confrontation des résultats issus des analyses pétroarchéologique (G. Porraz, P. Simon), technologique et typologique (G. Porraz) et tracéologique (A. Pasquini). Cette étude se structure à deux niveaux: la première échelle de l'analyse invite à caractériser le Proto-Aurignacien dans son identité technique et à en apprécier l'homogénéité à l'échelle de l'Europe occidentale; la seconde échelle de l'analyse invite à discuter de l'organisation territoriale de ces populations supposées «pionnières » et à apprécier leur structuration à un niveau socio-économique. Cette étude propose d'engager une discussion sur les rythmes et formes de diffusion du ProtoAurignacien, tels qu'ils sont perçus aujourd'hui à un moment et en un lieu de son histoire. L'objectif est donc d'explorer, à une échelle régionale, la réalité et les implications du paradigme de diffusion des hommes modernes en Europe en association avec le complexe proto-aurignacien.

\section{LA GROTTE DE L'OBSERVATOIRE}

\section{PRÉSENTATION GÉNÉRALE}

La grotte de l'Observatoire est située en bordure du rivage actuel de la mer Méditerranée, à Monaco (fig. 1). La grotte et les premiers vestiges de son occupation ont été mis au jour en 1916, à la suite de l'aménagement d'un promontoire rocheux (fig. 2). Le chanoine Léonce de Villeneuve, en collaboration avec Marcellin Boule, entreprit alors une fouille complète de l'ensemble du remplissage. Les résultats furent publiés dans le premier tome des Archives de l'Institut de Paléontologie humaine (1927) pour ce qui constitue, aujourd'hui encore, un remarquable témoignage scientifique. 


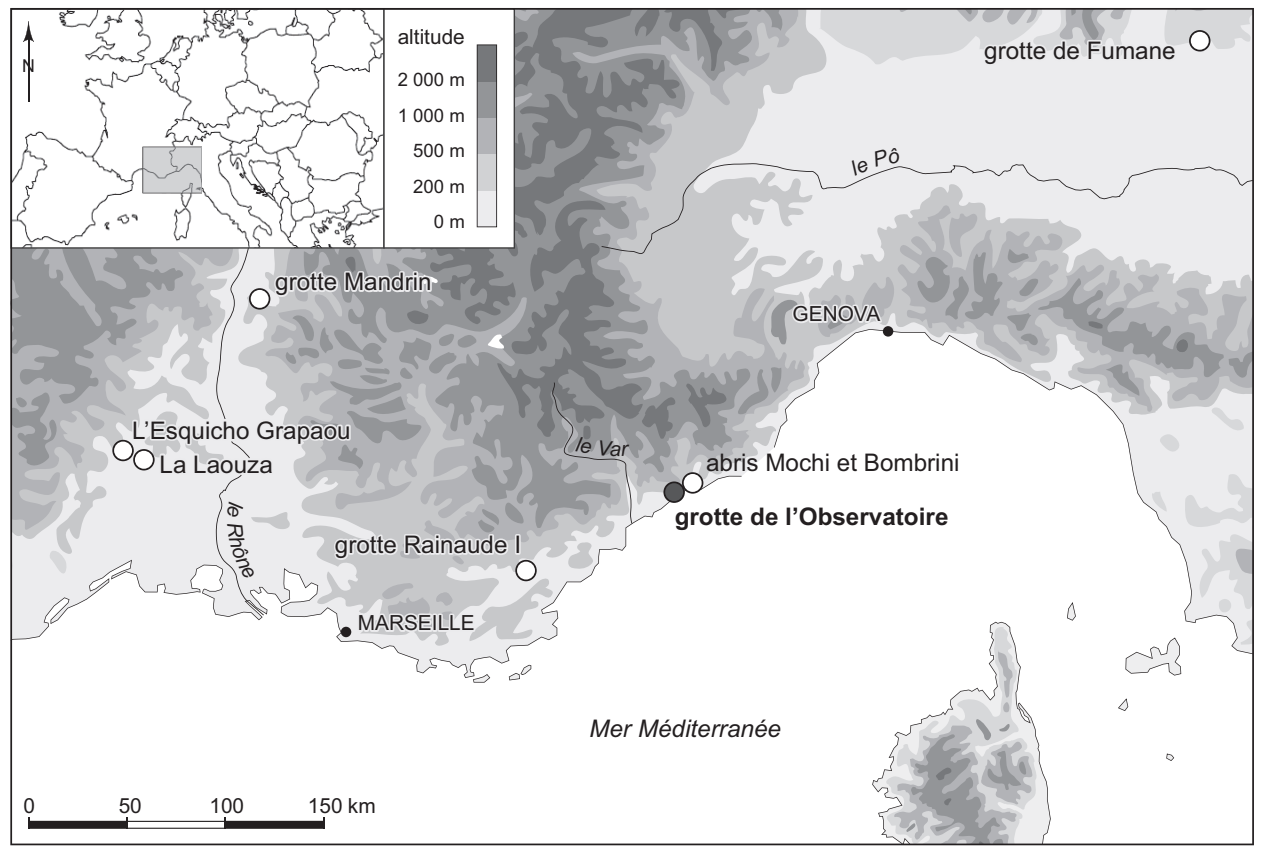

Fig. 1 - Localisation de la grotte de l'Observatoire (principauté de Monaco) et des sites proto-aurignaciens voisins (DAO: G. Porraz, Université de Tübingen).

La grotte, située face à la mer à une altitude de $104 \mathrm{~m}$, appartient aux formations calcaires du Jurassique supérieur. Elle se présente comme une grande anfractuosité liée à la présence d'une faille nord-est/sud-ouest à fort pendage sud-est. Le comblement quaternaire de la cavité, exploré dans sa partie supérieure sur près de $15 \mathrm{~m}$ de profondeur, a révélé la succession de trois ensembles stratigraphiques principaux (fig. 2). Ces ensembles, séparés par des planchers stalagmitiques (nouvelles datations en cours, V. Michel, P. Simon), distinguent trois phases d'occupation chronoculturelle larges:

- Phase I: la fosse, ou groupe inférieur du remplissage, livre les vestiges d'une tradition du Paléolithique inférieur. La présence de rares bifaces est ici associée à une production originale et systématique de gros éclats aux caractères morphométriques réguliers (étude en cours, G. Porraz, P. Simon).

- Phase II: la crypte, ou groupe moyen, contient des vestiges en faible nombre, diffus au sein de la séquence, attribués au Paléolithique moyen.

- Phase III: la chambre, ou groupe supérieur des dépôts, livre quant à elle des occupations de tradition du Paléolithique supérieur. L'ensemble des considérations et données présentées dans cet article se rapporte donc strictement à cette phase III.

\section{LES FOYERS G ET F}

C'est à la suite d'un réexamen des collections du Paléolithique supérieur qu'ont été mises en évidence, pour la première fois, des occupations proto-aurignaciennes au sein de la grotte de l'Observatoire (Onoratini et al., 1999). Ce sont plus précisément les foyers $\mathrm{G}$ et $\mathrm{F}$ qui ont livré une petite série lithique comprenant un lot caractéristique de lamelles Dufour (sous-type Dufour). Les comparaisons régionales, typologiques et technologiques ont alors appuyé les rapprochements de cette industrie proto-aurignacienne avec celles voisines de l'abri Mochi (couche G), de l'abri Bombrini (niveaux III) et de la grotte Rainaude (couche 10) (fig. 1; Onoratini, 2004; Onoratini, Simon, 2006).

Ces foyers $\mathrm{G}$ et $\mathrm{F}$, initialement attribués par M. Boule à l'Aurignacien moyen ou typique (au sens de H. Breuil), ont fait l'objet des descriptions suivantes:

«Le foyer G reposait en placage sur le grand plancher $\mathrm{n}^{\circ} \mathrm{I}$; il se montrait concrétionné comme lui. Nous n'avons pu le suivre que dans le tiers postérieur de la chambre. Plus haut, il se perdait au milieu de grosses pierres accumulées dans le pli médian du terrain (...). Au fond, le foyer $\mathrm{G}$ s'engageait dans un épais bourrelet stalagmitique, où se trouvaient pris un grand nombre de blocaux. Il n'y était reconnaissable qu'à quelques parcelles de charbon. 


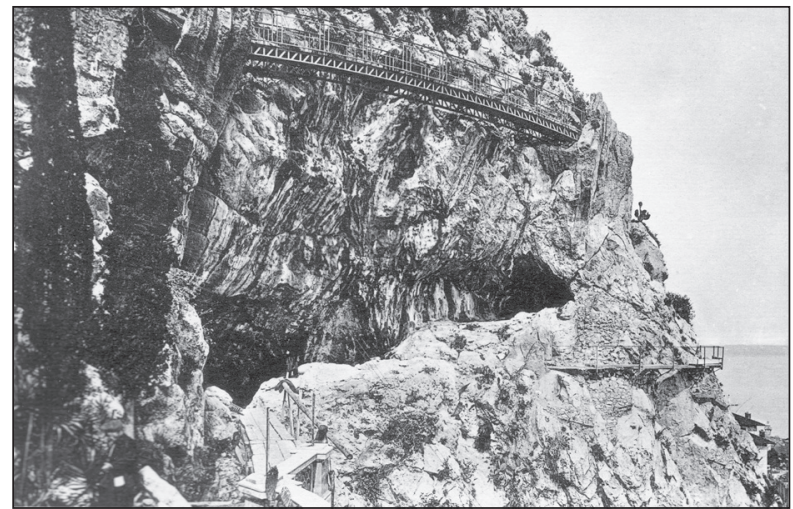

panoramique de la grotte de l'Observatoire (à gauche au premier plan)

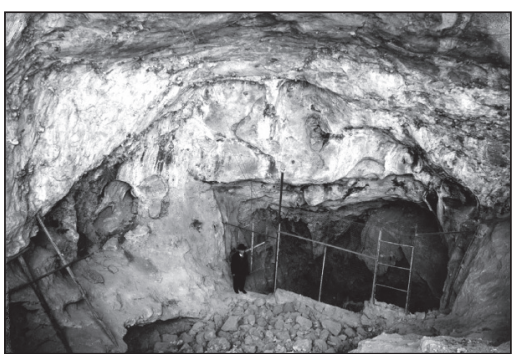

la Chambre après travaux
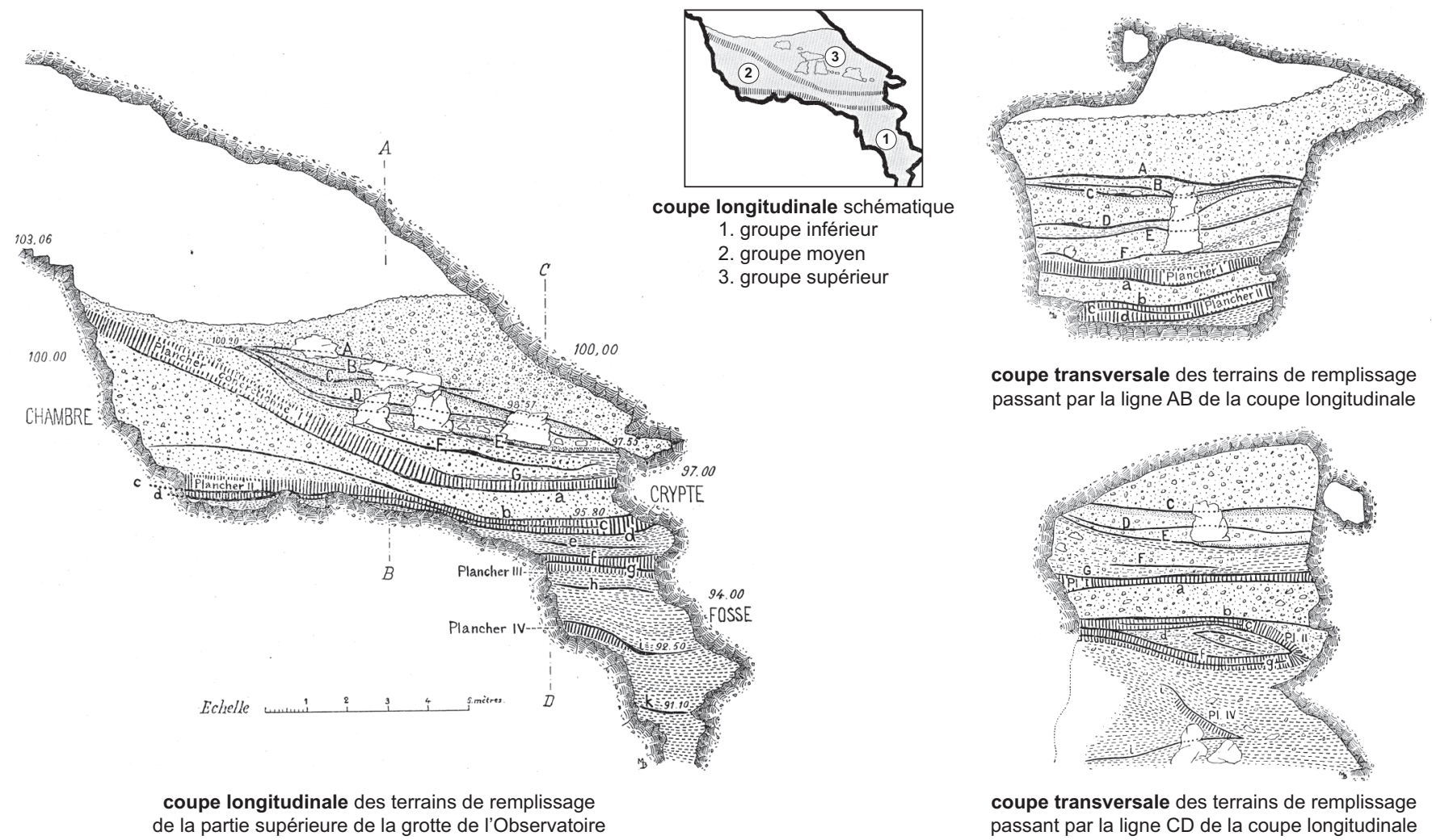

coupe transversale des terrains de remplissage passant par la ligne $A B$ de la coupe longitudinale

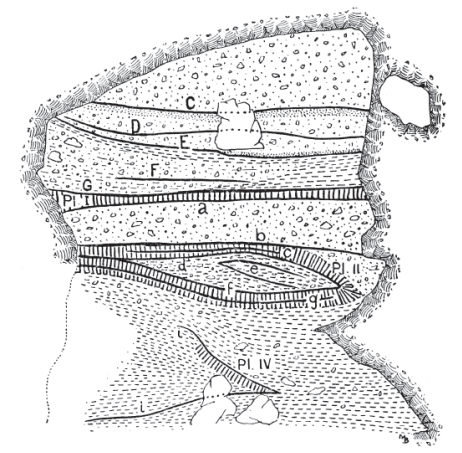

coupe transversale des terrains de remplissage passant par la ligne $C D$ de la coupe longitudinale

Fig. 2 - Documents d'archives de la grotte de l'Observatoire (d'après Boule, de Villeneuve, 1917).

Les hyènes y avaient passé. L'abondance des coprolithes en témoignait. Le foyer $G$ a fourni une tête de Cuon. D'importants restes de l'ours des cavernes, dont la portion faciale d'un crâne et quelques os longs, ont pu être dégagés (...). Une pointe en os, des grattoirs et quelques échantillons d'un outillage en silex prouvent que ce foyer appartient encore à la période archéologique aurignacienne»(Boule, Villeneuve, 1927, p. 18).
«Le foyer F (hauteur: 98,80 m). Sa nappe cinéritique, remaniée par le nivellement du dortoir, était presque partout rompue. Elle fut retrouvée à 5,50 m en avant du cul-de-four de la grotte, sous la forme d'un paquet de cendres débordant un amoncellement de grosses pierres. Sur la pente, quelques traces s'en révélèrent encore jusqu'à la rencontre d'un bourrelet de terre marneuse dont le niveau est inscrit sur le chevet de la chambre, un peu 
au-dessus de la cote 97. Cependant, au cours des travaux, le foyer $\mathrm{F}$ a été presque constamment indiqué par une ligne de teinte plus foncée que la masse du remplissage (...). Son existence ne laisse donc subsister aucun doute, d'autant moins que son interposition entre le foyer $\mathrm{E}$, que nous connaissons parfaitement, et le foyer $\mathrm{G}$, appliqué sur un plancher, ne nous aurait pas permis de nous égarer dans la restitution stratigraphique de sa marche discontinue et capricieuse» (Boule, Villeneuve, 1927, p. 17).

Les notes de terrain publiées dans la monographie de la grotte de l'Observatoire permettent de souligner deux points: d'une part, le sérieux avec lequel les fouilles ont été conduites et d'autre part, la complexité de l'agencement des dépôts dans la cavité, ce dont rendent compte les relevés stratigraphiques (fig. 2). De toute évidence, la morphologie du karst est à l'origine du fort pendage stratigraphique de cet ensemble supérieur, pendage que des phénomènes postdépositionnels (phénomènes de paroi, soutirages) ont dû raisonnablement accentuer. La sédimentation se trouve par ailleurs rompue par la présence de gros blocs d'effondrement, dont l'impact dynamique sur les dépôts n'est pas apprécié.

Les fouilles des dépôts supérieurs de la grotte de l'Observatoire ont consisté en la mise en lumière de foyers cendreux, organisant le découpage stratigraphique. Les dépôts, entièrement fouillés, constitués d'un limon rouge caillouteux, ont ainsi été subdivisés en sept unités stratigraphiques (de la base de la séquence à son sommet: foyers $G$ à A). À la lecture des descriptions de terrain, certains des foyers mis au jour semblent effectivement avoir constitué de vraies structures aménagées.

Compte tenu de l'ancienneté des fouilles et de la complexité des processus de sédimentation, l'intégrité stratigraphique de ces foyers se doit bien évidemment d'être discutée (voir infra, p. 38). Il convient toutefois de rappeler que la question de l'intégrité des foyers $\mathrm{G}$ et $\mathrm{F}$ ne se pose qu'à la lumière des dépôts sus-jacents. Le foyer $G$, premier ensemble du Paléolithique supérieur à la grotte de l'Observatoire, repose en effet sur un plancher stalagmitique (plancher I) l'isolant stratigraphiquement des occupations moustériennes antérieures.

«La couche concrétionnée, que nous appelons plancher I, forme, sur toute l'étendue du remplissage, une séparation hermétique entre l'étage supérieur et l'étage inférieur. Partant du seuil de la grotte à la cote 101,10, elle descendait presqu'en ligne droite sur les deux tiers de sa longueur et se continuait avec un redressement peu sensible vers le fond de la chambre, où elle se soudait à la cote 97. Composée (...) d'argile décolorée et de cailloux fortement agglutinés, par cela même fort résistante, cette couche épaisse de $30 \mathrm{~cm}$ et finalement réduite à $20 \mathrm{~cm}$ se dédoublait par le milieu, comme si elle avait été formée de deux lits d'apports exactement appliqués l'un sur l'autre » (Boule, Villeneuve, 1927, p. 18).

Le relevé et le marquage du matériel archéologique par unité stratigraphique ont été adoptés intégralement pour l'ensemble de l'industrie lithique. Les collections paléontologiques, quant à elles, ont été mélangées indistinctement et seules quelques rares annotations de terrain ou pièces remarquables autorisent aujourd'hui une remise en contexte. Le corpus faunistique identifié dans les foyers $\mathrm{G}$ et $\mathrm{F}$, à la lecture de la publication de 1927, regroupe assurément les espèces suivantes: Capra ibex, Cervus elaphus, Rhinoceros mercki, Cuon alpinus, Ursus spelaeus et Hyaena spelaea.

La question de l'attribution stratigraphique du matériel paléontologique soulève des problèmes dans le cadre d'une restitution large des économies de subsistance. Cette question est tout aussi délicate en ce qui concerne l'industrie osseuse. Si le foyer G contenait «une pointe en os», il demeure délicat d'en préciser la nature exacte. En l'état des connaissances, la majeure partie de l'industrie osseuse de la grotte de l'Observatoire est attribuée au foyer E:

«Ce même foyer E a livré, avec des coquilles percées d'un trou de suspension, plusieurs objets travaillés en os; ce seraient, d'après M. de Villeneuve, quelques pointes en os à base fendue du type d'Aurignac. Ces pièces figurent sur les planches XXV et XXVI, mais je n'ai pas les moyens de les séparer de celles qui proviennent d'autres foyers, sauf la plus grande, dite "lissoir" par M. de Villeneuve» (Boule, Villeneuve, 1927, p. 99).

La présence de sagaies à base fendue dans le foyer $\mathrm{E}$ rapporterait ce dernier à un Aurignacien ancien (ou Aurignacien I). Toutefois, la seule pièce clairement positionnée stratigraphiquement est un lissoir. Par ailleurs, les «coquilles percées d'un trou de suspension», qui ne sont pas sans évoquer celles découvertes dans les sites voisins de Mochi et Bombrini (Kuhn, Stiner, 1998; Negrino, 2006), n’ont pas été retrouvées. En tout état de cause, aucun élément de parure n'est associé aux foyers G et F de la grotte de l'Observatoire. 


\section{L'INDUSTRIE LITHIQUE DES FOYERS G ET F}

\section{DISCUSSION LIMINAIRE}

Les observations et notes de terrain permettent donc d'établir une distinction entre le foyer $\mathrm{E}$ (Aurignacien) et les foyers G et F (Proto-Aurignacien). Préalablement à l'analyse détaillée des collections, l'industrie lithique de la grotte de l'Observatoire a été observée dans sa totalité. Le matériel a été étudié foyer par foyer, puis regroupé par faciès lithologique. Le bon état général de conservation du matériel a alors facilité ces regroupements et notamment la réalisation des raccords, remontages et rapprochements, en premier lieu utilisés dans une perspective de reconstitution archéostratigraphique.

La question de l'intégrité des collections étudiées renvoie: aux processus dépositionnels et postdépositionnels, à la qualité des fouilles et des observations restituées, au traitement et au conditionnement postfouille, et à l'histoire muséale de la collection. L'ensemble des informations obtenues par l'étude des recoupements stratigraphiques relève par conséquent de cette genèse complexe. Dans un processus de restitution et de validation des ensembles archéostratigraphiques, la première enquête menée relevait, dans notre cas de figure, de considérations muséographiques ${ }^{1}$.

La projection sur un plan à une dimension des densités, raccords et remontages permet d'effectuer de premières observations (fig. 3). Tout d'abord, il s'avère que deux ensembles se démarquent numériquement: l'ensemble FG, correspondant au foyer $\mathrm{G}$, et l'ensemble EF, associé au foyer $\mathrm{E}$. Les foyers $\mathrm{G}$ et $\mathrm{E}$ seraient donc séparés par un foyer $\mathrm{F}$ relativement pauvre en vestiges lithiques (moins de 50 pièces), ce que corroborent les notes monographiques

1. Deux lots de pièces portent un marquage (lot FG, lot EF) à la signification équivoque (attribution à deux foyers ?). Dans les notes publiées ou conservées par M. Boule et L. de Villeneuve, aucun élément ne laisse envisager un tel enregistrement de terrain. La totalité de l'industrie lithique de la grotte de l'Observatoire, à l'origine, était classée et étiquetée par foyer. Ce matériel a fait l'objet d'un second conditionnement et d'un premier marquage dans le cadre du déménagement du musée, opération qui pourrait être à l'origine de la confusion soulevée. Au vu de la publication de 1927 et plus précisément de la planche XXI, il apparaît notamment que le lot marqué FG correspond à des pièces appartenant strictement au foyer $G$, et non aux foyers $\mathrm{F}$ et $\mathrm{G}$. Concernant le lot marqué $\mathrm{EF}$, nous considérons que celui-ci appartient essentiellement, sinon exclusivement, au foyer E, décrit dans la monographie comme un foyer "très riche» et qui, dans le cas contraire, ne serait alors représenté que par un effectif de pièces très réduit. parcimonieuses publiées à son sujet (Boule, Villeneuve, 1927).

Dans cette étude, le choix a été fait de regrouper les ensembles G, FG et $\mathrm{F}$ de façon univoque, regroupement motivé par la proximité des caractères technologiques et pétrographiques observés à un stade initial de l'étude. Pour autant, la distinction établie entre l'ensemble qui regroupe $\mathrm{G}$ et $\mathrm{F}$ et l'ensemble $\mathrm{E}$ ne doit pas non plus masquer les ressemblances observées du point de vue des industries lithiques. Ce choix d'étude s'est justifié par la nécessité de diviser l'assemblage lithique en ensembles cohérents, à défaut d'être stricts. En ce sens, l'industrie lithique du foyer E, associée à l'apparition de la parure et de l'industrie osseuse, devait être soustraite de cette première étude.

$\mathrm{Si}$ les raccords et remontages s'établissent majoritairement au sein d'un même ensemble stratigraphique (fig. 3), ils rendent néanmoins compte de certains recouvrements. L'ampleur des interstratifications observées s'avère toutefois limitée ${ }^{2}$, un unique raccord témoignant de bouleversements importants (foyer G-foyer A). Par ailleurs, les nombreux rapprochements effectués, non projetés en figure 3 , confirment globalement la cohérence des ensembles stratigraphiques considérés.

\section{PRÉSENTATION GÉNÉRALE}

\section{ÉTAT DE CONSERVATION}

Du point de vue de l'étude pétroarchéologique, les altérations peuvent être considérées comme peu prononcées. Les altérations physico-chimiques des silex soulignent des processus très localisés au sein de la cavité, ce qu'illustrent plusieurs remontages d'éclats aux altérations de surface différentielles (patines). Les matières premières siliceuses des niveaux $\mathrm{G}$ et $\mathrm{F}$ de la grotte de l'Observatoire ont ainsi fait l'objet d'attributions pétroarchéologiques dans près de $85 \%$ des cas de figure. Ces caractérisations, préliminaires, ont été conduites à la loupe binoculaire (x 10/x 40); les attributions se sont appuyées sur les référentiels géologiques de comparaison déposés au musée d'anthropologie de Monaco et au laboratoire du CEPAM/CNRS à Valbonne.

2. Les deux pièces de l'ensemble $\mathrm{G}$ qui remontent avec des pièces du foyer E (fig. 3) appartiennent en réalité à un petit lot marqué G0. La note associée à cet ensemble G0 est la suivante: "Les éclats ont été trouvés dans une poche de fond de la chambre à la cote 95,80 environ et par conséquent au-dessous du grand plancher; mais leur contact avec la paroi rocheuse laisse à penser qu'ils y ont glissé d'un niveau supérieur par un intervalle entre la roche encaissante et la surface concrétionnée.» 


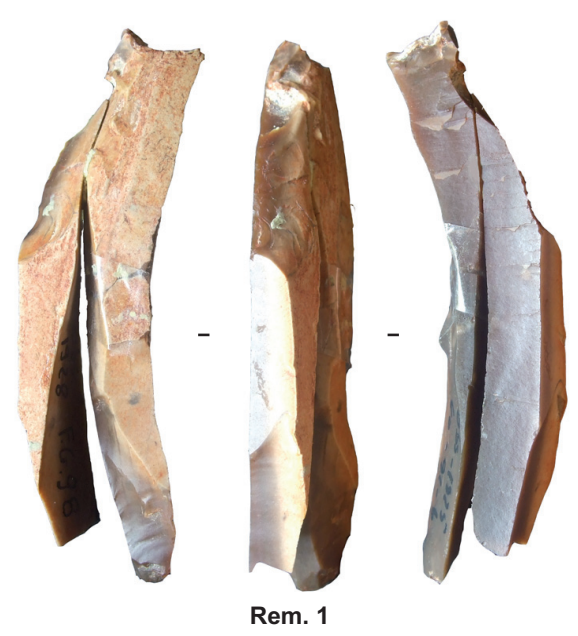

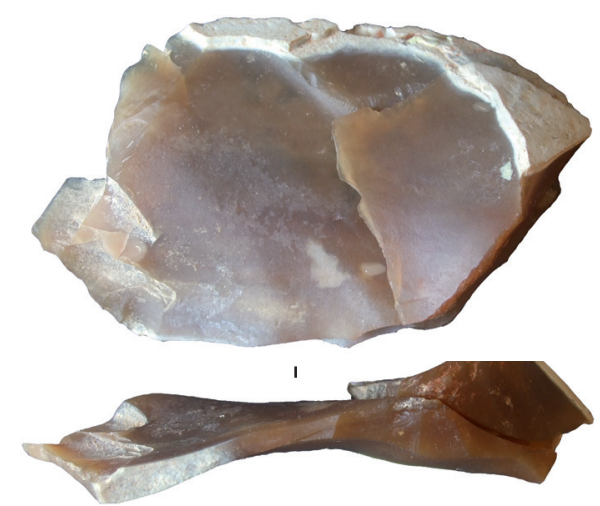

Rem. 2

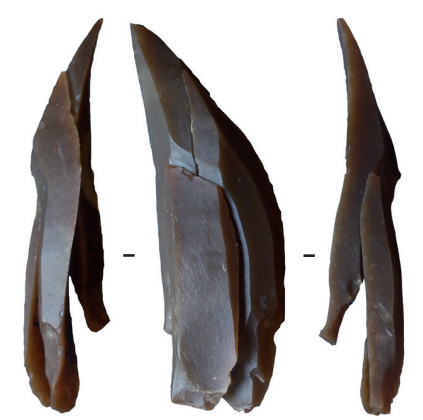

Rem. 3

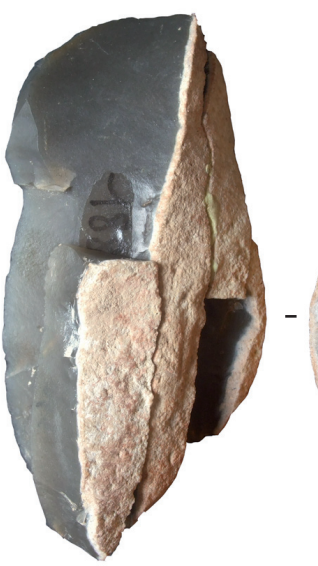

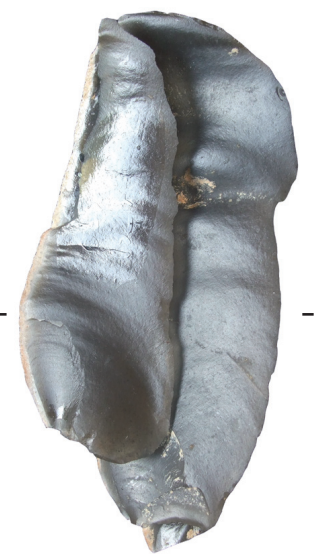

Rem. 4

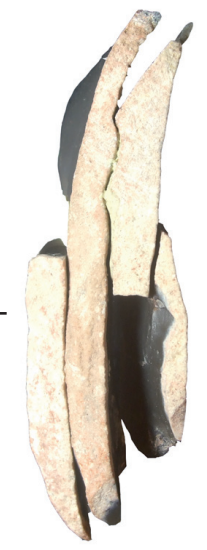

0

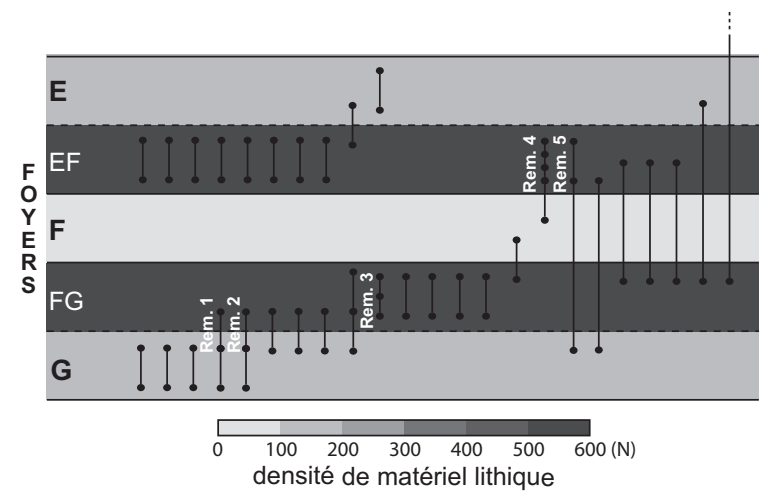

Les indéterminables sont représentés par des individus aux états d'altération trop importants (pièces patinées ou brûlées) et les indéterminés par de plus rares éléments non reconnus, soit qu'ils s'inscrivent dans une variabilité de faciès non cernée, soit qu'ils restent à ce jour sans concordance au sein des collections géologiques de référence.
Fig. 3 - Projection à une dimension des raccords et remontages lithiques des foyers $G, F$ et $E$ de la grotte de l'Observatoire (DAO: G. Porraz, Université de Tübingen).

Les choix de présentation volontairement adoptés dans cet article abordent la variabilité pétroarchéologique dans ses tendances, et non dans ses détails.

L'étude tracéologique s'est confrontée à des limites d'analyse plus contraignantes (luisance, esquillements de manipulation, etc.). Cette étude a comporté une première 
expertise à l'œil nu, suivie par la recherche des macrotraces à faible grossissement et des microtraces à fort grossissement (pour les pièces considérées diagnostiques à ces premières échelles d'observation). L'analyse fonctionnelle n’a négligé aucune des catégories technotypologiques et pétrographiques.

Une partie importante du matériel (environ $20 \%$ ) porte les traces nettes ou résiduelles d'un dépôt ocré. La répartition de cet «ocre» sur le matériel lithique est, sinon aléatoire, du moins très hétérogène; elle est en partie artificielle lorsque l'on considère les manipulations et conditionnements successifs de la collection. Ces colorants se retrouvent naturellement mieux conservés dans les parties les plus irrégulières (parties corticales, terminaisons de négatifs: c'est-à-dire des parties abrasées ou retouchées). Quelques pièces toutefois présentent des organisations de plages ocrées qui invitent à rechercher des causes et des origines combinées (surfaces ocrées recoupées d'un ou de plusieurs négatifs d'enlèvements). Au final, si l'association de cet ocre avec l'industrie lithique est à première vue fortuite, elle n'en est pas moins indiscutable.

\section{COMPOSITION GÉNÉRALE}

L'industrie lithique des foyers $\mathrm{G}$ et $\mathrm{F}$ regroupe environ 750 pièces (133 pièces proviennent de l'ensemble G, 572 de l'ensemble FG, 43 de l'ensemble F). Le décompte technologique global rend compte d'une nette sous-représentation des éléments de dimension infracentimétrique (tabl. I). Cette observation, vérifiée sur l'ensemble de la séquence, est à mettre directement en rapport avec les normes de fouille adoptées à l'époque. Suivant ce constat, la question de la représentation effective de la petite production lamellaire doit être soulevée. Toutefois, l'observation des nucléus lamellaires à leur stade d'abandon et leur confrontation avec les gabarits de lamelles retrouvées ne permettent pas d'envisager une forte distorsion de la réalité archéologique.

L'attribution première des foyers $\mathrm{G}$ et $\mathrm{F}$ au ProtoAurignacien repose sur un lot de lamelles Dufour, sous-type Dufour ( $n=23)$, bien représentées au sein de l'échantillon typologique (tabl. II). La distinction établie a posteriori entre les lames et lamelles repose précisément sur l'observation des Dufour. Leur étude permet ainsi d'individualiser une population de lamelles de largeur inférieure ou égale à $10 \mathrm{~mm}$, limite supérieure qui a donc été adoptée et généralisée à l'ensemble de l'étude.
Tabl. I - Décompte par catégorie technologique de l'industrie lithique des foyers $G$ et $F$ de la grotte de l'Observatoire.

\begin{tabular}{|l|c|c|}
\hline \multicolumn{1}{|c|}{ Catégorie } & Effectif (N) & Effectif (\%) \\
\hline Éclat & 127 & 17 \\
\hline Lame & 208 & 28 \\
\hline Lamelle & 226 & 30 \\
\hline Nucléus & 14 & 2 \\
\hline Tablette & 75 & 10 \\
\hline Éclat $<20 \mathrm{~mm}$ & 27 & 3,5 \\
\hline Débris et fragment & $\mathbf{7 2}$ & 9,5 \\
\hline Total & $\mathbf{7 4 8}$ & $\mathbf{1 0 0}$ \\
\hline
\end{tabular}

Tabl. II - Décompte typologique de l'industrie lithique des foyers $G$ et $F$ de la grotte de l'Observatoire.

\begin{tabular}{|l|c|c|}
\hline \multicolumn{1}{|c|}{ Typologie } & Effectif $(\mathbf{N})$ & Effectif $(\%)$ \\
\hline Lamelle Dufour & 23 & 29 \\
\hline Lamelle retouchée & 12 & 15 \\
\hline Lame retouchée & 22 & 29 \\
\hline Lame encochée & 6 & 7 \\
\hline Burin & 10 & 12 \\
\hline Grattoir & 7 & 8 \\
\hline Total & $\mathbf{8 0}$ & $\mathbf{1 0 0}$ \\
\hline
\end{tabular}

\section{LES CHAÎNES OPÉRATOIRES DE PRODUCTION LITHIQUE}

Les supports laminaires et lamellaires constituent les deux principaux objectifs de production. Ils ont en commun de témoigner de phases d'acquisition sélectives orientées vers des matériaux de très bonne qualité, d'être issus d'un schéma de production unipolaire et de présenter un profil peu courbe à rectiligne.

La reconstitution des chaînes opératoires repose ici sur la lecture d'un assemblage lithique composé de multiples événements. L'estimation élevée du nombre minimum de blocs (NMB) appuie l'impression d'une importante distorsion techno-économique des activités dans l'espace, distorsions pour partie liées à l'éloignement des sources de matières premières exploitées. L'analyse des chaînes opératoires se fonde donc sur la lecture de courtes séquences de production, ce que confirme l'empreinte générale des remontages et rapprochements composés d'un faible nombre d'éléments (tabl. III). Au final, le panel des «séquences" effectivement conduites ou reconstituées autorise toutefois une bonne appréciation des objectifs et règles de production. 
Tabl. III - Décompte lithotechnologique de l'industrie lithique des foyers $G$ et $F$ de la grotte de l'Observatoire.

\begin{tabular}{|c|c|c|c|c|c|c|c|c|c|c|c|}
\hline & Éclat & Lame & \begin{tabular}{|c|} 
Lamelle \\
(dont Dufour) \\
\end{tabular} & Nucléus & Tablette & \begin{tabular}{c|} 
Éclat \\
$<20 \mathrm{~mm}$ \\
\end{tabular} & Débris & $\begin{array}{l}\text { Total } \\
\text { (N) }\end{array}$ & $\begin{array}{l}\begin{array}{c}\text { Total } \\
(\%)\end{array} \\
\end{array}$ & Rem.* & NMB* \\
\hline Local $(\approx 10 \mathrm{~km})$ & & & & & & & & 7 & 1 & & \\
\hline Silex de « I Ciotti » & 5 & & 1 & & & & 1 & 7 & & 0 & 4 \\
\hline Faciès de Provence orientale (> $40 \mathrm{~km}$ ) & & & & & & & & 45 & 7 & & \\
\hline Kimméridgien-Portlandien & 3 & 4 & 3 & 5 & 3 & 1 & 1 & 20 & & 0 & 6 \\
\hline Hauterivien-Valanginien & & & 3 & & & & & 3 & & 0 & 1 \\
\hline Éocène & & 5 & 2 & & 1 & & 1 & 9 & & 0 & 6 \\
\hline Sannoisien & 4 & 5 & 3 & & 1 & & & 13 & & $1 \times 2$ & 2 \\
\hline Faciès de Provence occidentale (> $130 \mathrm{~km})$ & & & & & & & & 542 & 87 & & \\
\hline Bédoulien & 39 & 37 & $50(7)$ & 1 & 18 & 7 & 10 & 162 & & 4 & 11 \\
\hline Oligocène & 60 & 112 & $116(13)$ & 7 & 45 & 12 & 29 & 381 & & $5 \times 2 / 3 \times 3$ & 21 \\
\hline Faciès italiens $(200-300 \mathrm{~km})$ & & & & & & & & 26 & 4 & & \\
\hline Jaspe & & 2 & 1 & & & & 1 & 4 & & 0 & 1 \\
\hline Silex « à calpionelles » & 6 & 4 & $6(1)$ & & 1 & 3 & & 20 & & 0 & 3 \\
\hline Scaglia Marchigiana & & 1 & 1 & & & & & 2 & & 0 & 2 \\
\hline Indéterminé, non attribué & 9 & 38 & $40(2)$ & 1 & 6 & 4 & 29 & 127 & & & 2 \\
\hline Total & 126 & 208 & $226(23)$ & 14 & 75 & 27 & 72 & 748 & - & $10 \times 2 / 3 \times 3$ & 59 \\
\hline
\end{tabular}

Rem. : nombre de remontages $x$ nombre d'éléments remontés ; NMB : nombre minimum de blocs

\section{ACQUISITION DES MATIÈRES PREMIÈRES}

Les matières premières identifiées dans les foyers $G$ et $\mathrm{F}$ de la grotte de l'Observatoire peuvent être divisées en quatre principaux groupes ou espaces économiques d'approvisionnement. Ces espaces sont toutefois inégalement représentés, puisque les silex des formations carbonatées de Provence rhodanienne composent près de $85 \%$ du corpus lithologique déterminé (tabl. III) (fig. 4).

\section{GROUPE 1 : LES FACIÈS DU CONGLOMÉRAT DE $\ll$ I CIOTTI»}

Les environs immédiats de la grotte de l'Observatoire sont dépourvus de matériaux de bonne qualité à la taille. Les disponibilités en matières premières locales sont à rechercher plus à l'est, à la frontière franco-italienne, précisément à l'emplacement des sites du complexe des Balzi Rossi. Des galets de silex, issus du démantèlement du conglomérat éocène de «I Ciotti ", peuvent donc être récoltés à une distance approximative de $10 \mathrm{~km}$ à l'est depuis la grotte.

Ces silex, dont la cassure est irrégulière, présentent différents faciès qui ont en commun d'être très cristallisés. Ils sont opaques à semi-translucides, de couleur gris-beige à brun, de structure généralement massive, plus rarement litée. Ces silex et calcaires siliceux témoignent dans leur ensemble de qualité, à la taille, très moyenne.
Les matériaux provenant du conglomérat de «I Ciotti » ne sont que très faiblement représentés dans les niveaux proto-aurignaciens de la grotte de l'Observatoire. Seuls cinq éclats et une lamelle témoignent ainsi de l'exploitation très épisodique de ces matières premières locales, pour un NMB estimé à 4 .

\section{Groupe 2: LES FACIÈS DE PROVENCE ORIENTALE}

La séquence sédimentaire de la Provence orientale livre des silex en quasi-continuité depuis les formations du Trias jusqu'à celles de l'Oligocène. Les disponibilités sont toutefois inégales sur l'ensemble de ce secteur et ce sont plus précisément les fossés tertiaires nord-varois et les formations voisines du Jurassique supérieur qui livrent les silex de meilleure qualité (Binder et al., 1994; Porraz, 2005).

Des silex appartenant aux formations du KimméridgienPortlandien ont pu être identifiés à la grotte de l'Observatoire. Il s'agit de silex gris-blanc, au cortex blanc à roux millimétrique, carbonatés, de structure massive, parfois zonés, dans certains cas très riches en bioclastes (spicules, foraminifères, brachiopodes). Ces silex se présentent sous la forme de nodules ovoïdes ou branchus, de dimensions centimétriques à pluri-décimétriques. Ces matériaux, souvent diaclasés, peuvent toutefois présenter une excellente aptitude à la taille (Porraz et al., 2009).

Ces silex du Jurassique supérieur sont représentés par un lot de vingt pièces, composé d'un nombre important de 


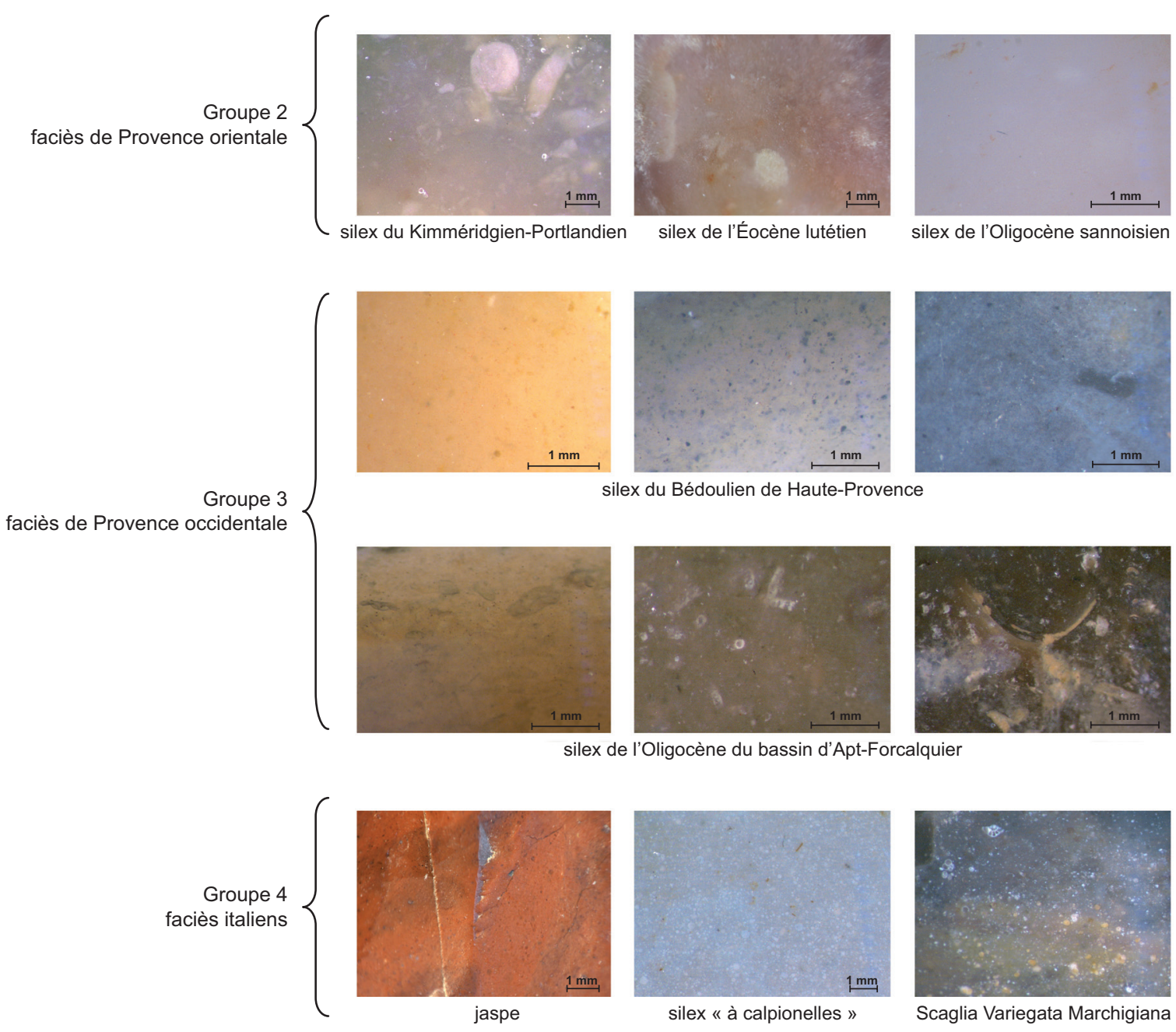

Fig. 4 - Vues macroscopiques des faciès pétrogéographiques individualisés à la grotte de l'Observatoire (DAO: G. Porraz, Université de Tübingen).

nucléus à lamelles $(\mathrm{n}=5)$. Les cortex attestent la récolte de blocs en position subprimaire, pour des distances de transport minimales de $40 \mathrm{~km}$ à l'ouest (plateaux de Caussols et de Calern, montagne de l'Audibergue). Ce lot est constitué d'un NMB évalué à 6 .

Certains silex proviennent des fossés nord-varois. Ce sont d'abord les silex gris-bleu à vert des formations de l'Hauterivien-Valanginien qui peuvent être individualisés. Ces matériaux, très fins, de structure massive, associent notamment la présence de petits quartz détritiques et des fragments de petite coquille de bivalve. Ces silex sont géographiquement bien circonscrits et constituent de très bons matériaux traceurs. À la grotte de l'Observatoire, ces matières premières ne sont que très faiblement représentées puisque seules 3 lamelles sont attribuées à ces formations.
Ils proviennent d'un secteur distant d'environ $60 \mathrm{~km}$ à l'ouest de la grotte de l'Observatoire.

Les silex éocènes provenant de ce même secteur ne sont que faiblement représentés. Il s'agit d'éléments isolés $(\mathrm{n}=9)$ qui permettent de proposer une estimation haute du NMB, évalué à 6 . Plusieurs variétés peuvent ainsi être distinguées sur la base de leur couleur, de leur structure, de leur détritisme et de leur contenu litho- et bioclastique.

Les silex oligocènes des formations du Sannoisien sont pour leur part facilement individualisables. Il s'agit de matériaux translucides, blancs «floconneux», de texture mudstone, peu détritiques, à cortex blanc ou jaune poreux. Ces matériaux, introduits sous forme de plaquette ou d'éclat, rassemblent treize éléments pour un NMB de 2. L'estimation du NMB repose ici sur des critères techno- 
logiques avec l'individualisation a minima d'un débitage sur plaquette et d'un débitage sur gros éclat.

\section{Groupe 3 : LES FACIÈS DE PROVENGE OCGIDENTALE}

Les analyses pétroarchéologiques attribuent un rôle prééminent aux silex de Provence occidentale: les silex du Crétacé inférieur et de l'Oligocène (Binder et al., 1998) composent ainsi plus de $85 \%$ du corpus lithologique identifié (20 lamelles Dufour sur 21 déterminées).

Les silex du Crétacé inférieur, plus précisément des formations du Bédoulien, constituent plus de $25 \%$ de la totalité du matériel déterminé. Le silex dit «blond», de couleur miel, constitue le microfaciès le mieux représenté. Il s'agit d'un silex très homogène, à pâte saumonée, à petits quartz détritiques, peu bioclastique (pellets, spicules). Un faciès gris, un faciès blond, un faciès rosé, un faciès grisbleu translucide et un faciès gris-bleu opaque permettent d'introduire un premier niveau de variabilité. La couleur de pâte, la présence de zones carbonatées, l'abondance des quartzs détritiques ou encore les types et fréquences des bioclastes (foraminifères) permettent donc d'individualiser différents microfaciès et zones de provenance. L'ensemble des observations effectuées pointent le domaine de HauteProvence, incluant les régions des monts de Vaucluse, du mont Ventoux et de la montagne de Lure, comme lieu d'origine de ces matières premières (de $130 \mathrm{~km}$ à $180 \mathrm{~km}$ à l'ouest). Les cortex observés sur ces matériaux attestent une récolte de blocs effectuée pour partie en position subprimaire. Le travail de caractérisation en cours devrait, à terme, permettre une individualisation plus fine des lieux et sources de provenance, ainsi qu'une estimation plus exacte du NMB $(\mathrm{NMB}=10)$.

Les silex lacustres de l'Oligocène constituent pour leur part plus de $60 \%$ de la totalité du matériel déterminé. Les différents microfaciès individualisés au sein de cet ensemble trouvent leur origine au sein du bassin d'Apt-Forcalquier (environ $130 \mathrm{~km}$ à l'ouest). Il s'agit de silex de couleur brun à noir, de structure massive à litée, certaines altérations révélant une structure caractéristique «xyloïde» ou «rubanée». Ces matériaux se distinguent par leur texture (mudstone à wackestone) ainsi que par leur contenu et association bioclastique. L'état de conservation et l'abondance des tiges de characées, oogones, fragments de coquilles de bivalves et ostracodes, ont permis d'établir certains rapprochements et, à l'inverse, de dépareiller certains éléments. Les cortex associés à ces faciès oligocènes attestent une récolte de plaquettes et blocs en position subprimaire.
L'estimation préliminaire du NMB permet de proposer une estimation basse de 20 blocs.

\section{GROUPE 4 : LES FACIÈS ITALIENS (LIGURIE, EMILIE-ROMAGNE, MARCHES)}

Les matériaux provenant de secteurs situés plus à l'est de la grotte de l'Observatoire sont, de façon étonnante, assez peu représentés puisqu'ils regroupent environ $4 \%$ des matériaux déterminés ${ }^{3}$.

Un petit lot de pièces en jaspe ${ }^{4}(\mathrm{n}=4)$ provient des formations du Jurassique supérieur des environs de Monte Lama et de Monte Pràrbera, en Ligurie orientale et Emilie-Romagne. Ces jaspes, bien documentés dans la littérature, ont été introduits sous forme de produits finis et témoignent de distances d'approvisionnement de l'ordre de 200 km (Del Soldato et al., 1987; Del Soldato, 1990; Negrino, Starnini, 2003).

Un lot de silex gris-blanc "perlé», dont la couleur est parfois légèrement violacée à verdâtre, ressemble fortement aux matériaux récoltés dans les formations des calcaires à calpionelles des Apennins (Emilie-Romagne). Ces silex, de très bonne qualité et caractérisés par la présence de radiolaires, proviendraient de sources distantes a minima de $200 \mathrm{~km}$.

Deux pièces en silex proviennent des formations de la Scaglia Variegata Marchigiana. L'une d'entre elles, de couleur gris-bleu et au cortex blanc poreux, témoigne d'un prélèvement en position subprimaire. Ce faciès, particulièrement riche en foraminifères planctoniques (notamment Subbotina sp., voir Cancellieri et al., à paraître) et déjà identifié dans d'autres sites proto-aurignaciens voisins (Negrino, Starnini, 2003), témoigne d'un transport effectué sur des distances supérieures à $400 \mathrm{~km}$.

\section{ORGANISATION DE LA PRODUCTION}

La nature des produits abandonnés dans la cavité permet d'abord de bien apprécier les principes de la production lamellaire. Le schéma premièrement identifié

3. Nous remercions chaleureusement Fabio Negrino (Université de Pise) pour ses remarques et observations, ainsi que pour les nombreux échantillons géologiques mis à disposition. Nos remerciements s'adressent également à Marco Peresani et Emanuele Cancellieri (Université de Ferrara) pour nous avoir communiqué les premiers résultats de leurs travaux sur les silex de la Scaglia Marchigiana et pour nous avoir fait suivre de nombreux échantillons.

4. Il s'agit à proprement parler de radiolarite mais nous conservons ici le terme de «jaspe» consacré dans la littérature. 

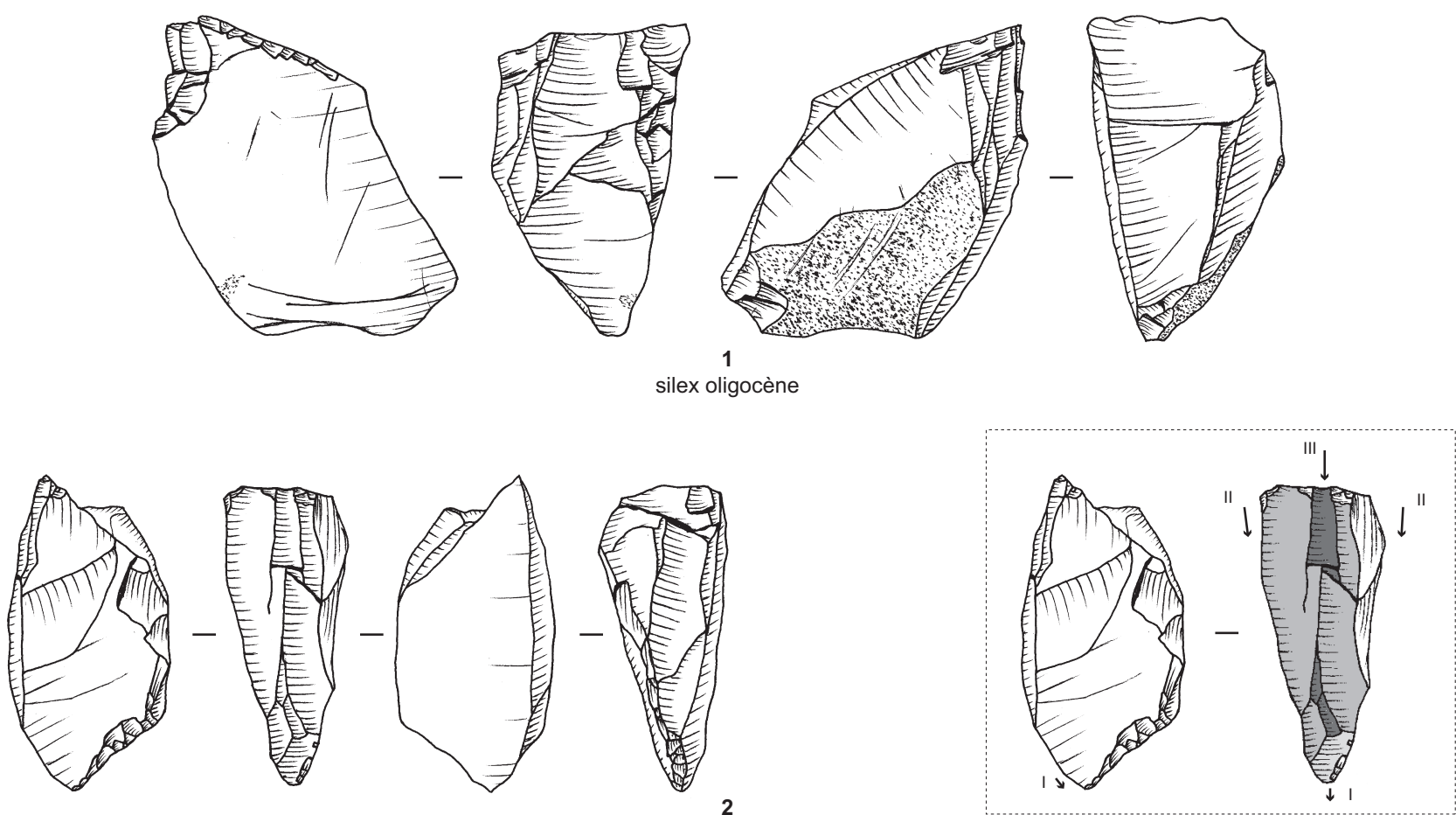

silex kimméridgien-portlandien
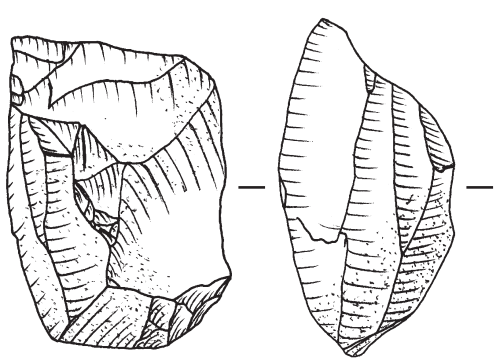

3

silex bédoulien

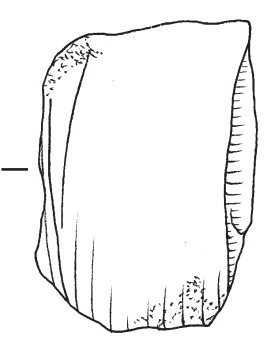

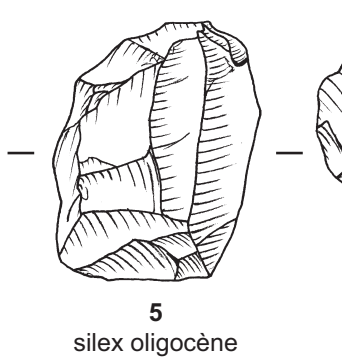

silex oligocène
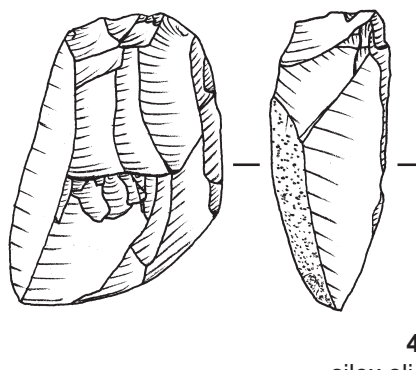

4

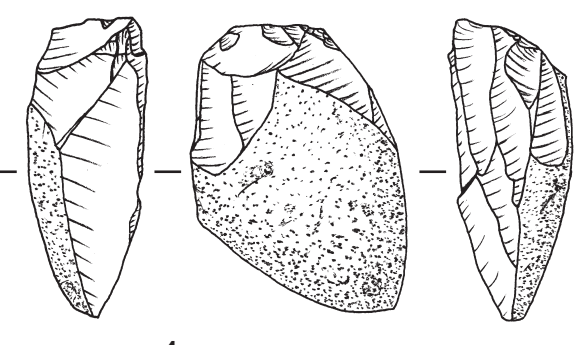

silex oligocène

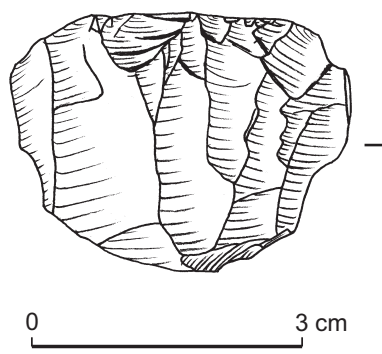

Fig. 5 - Nucléus lamellaires du Proto-Aurignacien de la grotte de l'Observatoire: 1-3, sur éclat (1-4: foyer FG; 5: foyer F) (dessin: G. Porraz, Université de Tübingen). débute par une sélection de gros éclats en vue d'une exploitation de leur volume étroit (fig. 5, $\mathrm{n}^{\mathrm{os}} 1-3$ ). L'initialisation de la production se fait par la réalisation d'une crête à un versant, complète ou partielle, réalisée systématiquement aux dépens de la face supérieure de l'éclat-support (fig. 6, $\left.\mathrm{n}^{\text {os }} 1-3\right)(\mathrm{n}=9)$.
Le contrôle de la production est alors assuré par le détachement d'enlèvements enveloppants (contrôle de la carène), de flanc (contrôle du cintre) et centrés (voir fig. 5 , $n^{\circ} 2$ ). Cet agencement du débitage, en trois temps, détermine une production relativement normée. Celle-ci est composée de lamelles courbes au profil distal «brisé », de 

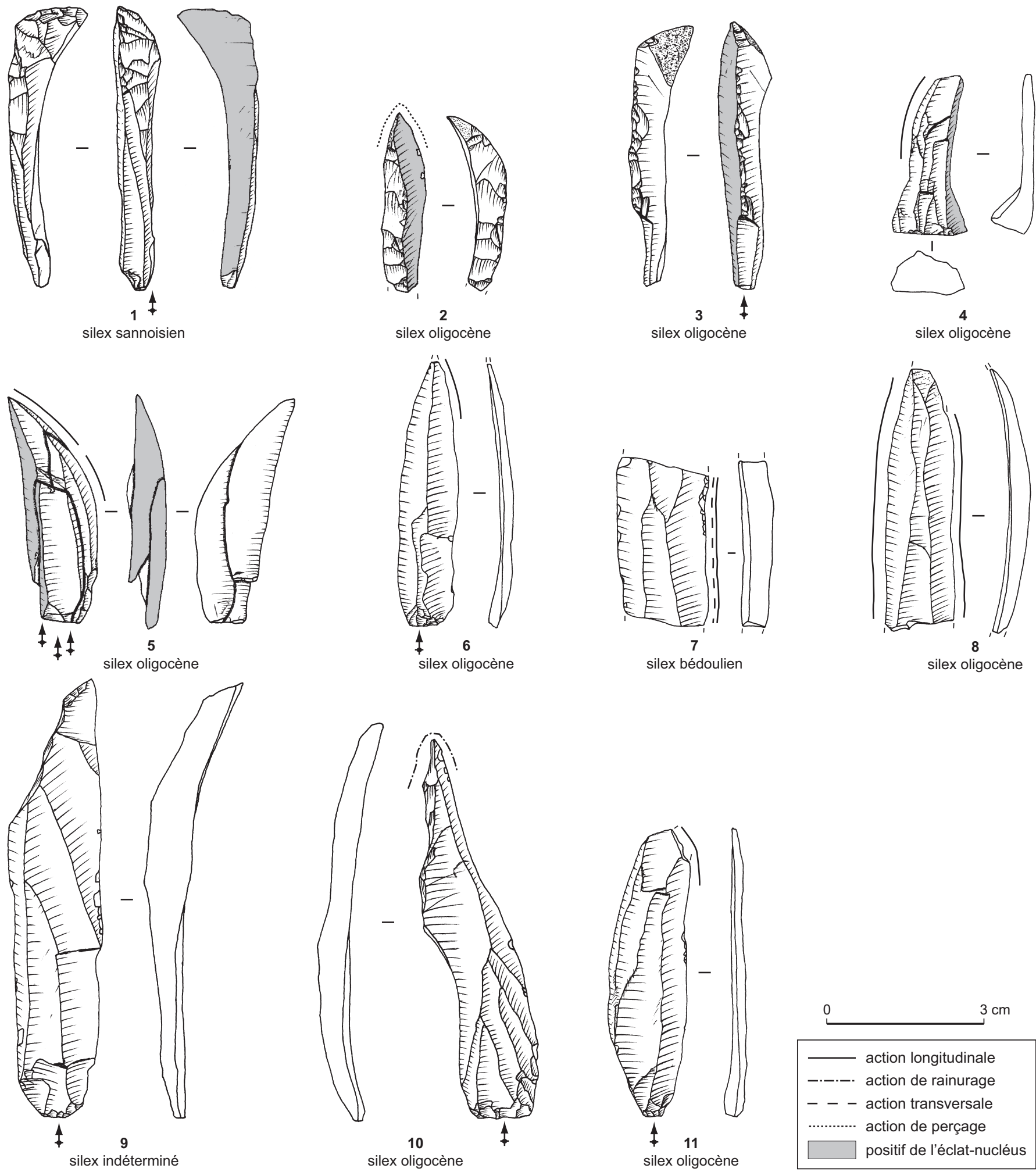

Fig. 6 - Produits lamellaires et laminaires du Proto-Aurignacien de la grotte de l'Observatoire: 1-3, lamelles à crête à un versant; 4, lamelle présentant un accident de taille proximal dû à la présence d'une diaclase libérée lors de la percussion; 5 , remontage de 3 produits lamellaires comprenant l'intercalation d'une réfection du plan de frappe; 6-8, 11, lames de plein débitage; 9, 10, lames néo-crête (1-5, 7: foyer FG; 6, 9, 11 : foyer $G$; 8, 10: foyer F) (dessin: G. Porraz, Université de Tübingen). 

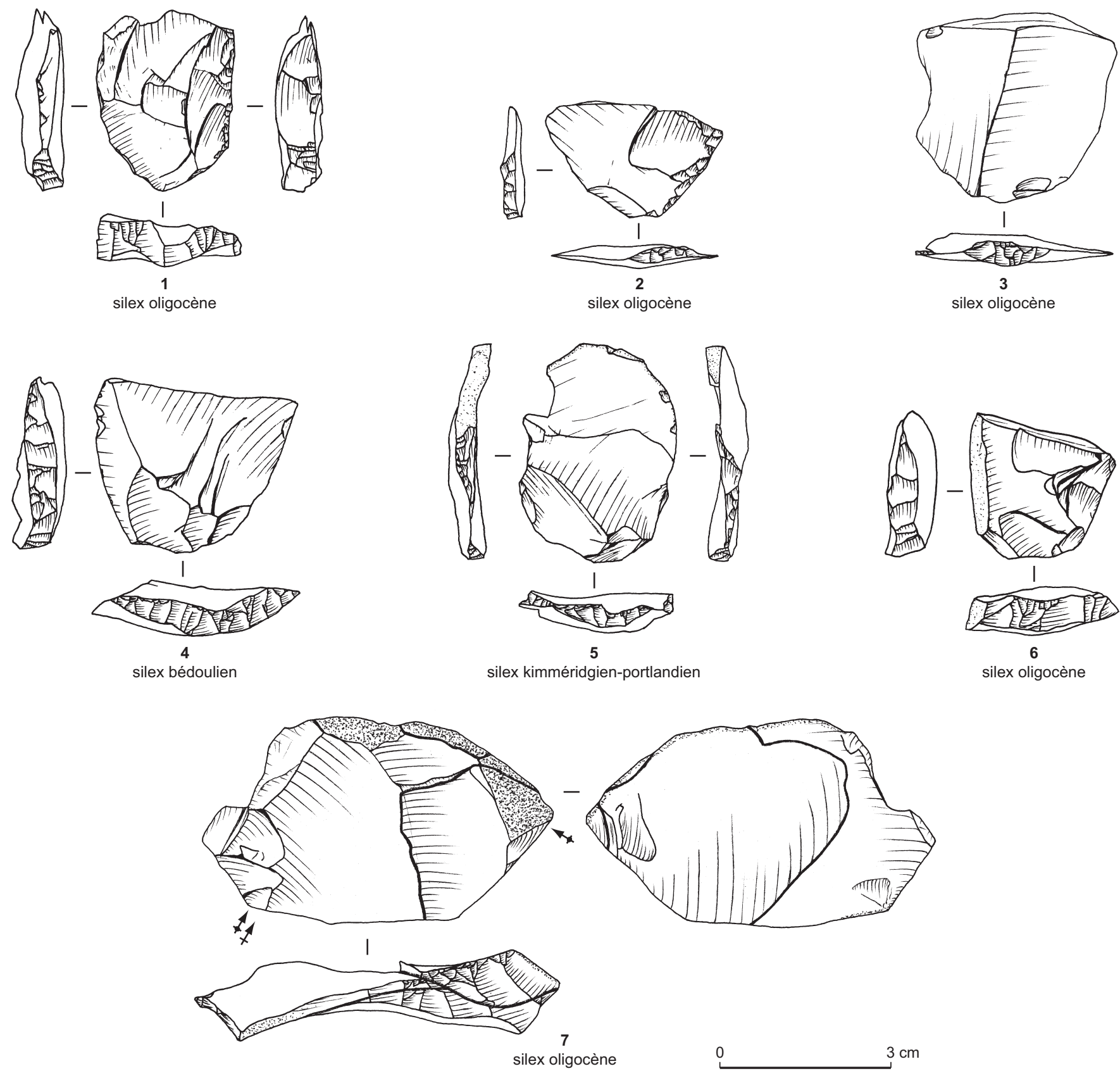

Fig. 7 - Produits de réfection des plans de frappe des nucléus proto-aurignaciens de la grotte de l'Observatoire: 1-4, 6, tablettes partielles; 5, tablette totale; 7, remontage de trois tablettes $(\mathbf{1}:$ foyer $F ; 2,4,5:$ foyer $F G ; 6$ : foyer $G ; 7:$ foyers $F G$ et $G)$ (dessin: G. Porraz, Université de Tübingen).

lamelles latérales pouvant présenter un positif de l'éclatnucléus, et enfin de lamelles centrées très régulières aux nervures parallèles ou convergentes. Il convient par ailleurs de mentionner la présence de rares lamelles à négatifs croisés renvoyant à un schéma de production dans lequel l'aménagement du volume débité est assuré par le déta- chement d'enlèvements lamellaires obliques vis-à-vis de la surface d'exploitation (Slimak et al., 2006).

Cet agencement du débitage paraît commun aux productions lamellaires et laminaires. Peu d'informations sont directement disponibles concernant les phases d'initialisation du débitage laminaire. Un remontage (fig. 3, 
rem. 1) témoigne de l'exploitation du volume étroit d'une plaquette, après aménagement d'une crête à un versant. Ce schéma d'initialisation, dans le cadre d'une production de petites lames et grandes lamelles, identifié sur d'autres matériaux, est dans ce cas très proche de celui identifié pour la production lamellaire sur éclat.

Ce schéma de production en volume étroit sur éclat ou plaquette est associé à un schéma de production sur blocs l.s. exploités dans leur volume large. Au vu des produits disponibles, seul le cintrage du nucléus semble alors faire l'objet de préparations spécifiques (réalisation de néocrêtes latérales, voir fig. $6, \mathrm{n}^{\text {os }} 9$ et 10) $(\mathrm{n}=6)$. Quelques grandes lames et fragments de lame (fig. 6), témoignant de productions très soignées, proviennent vraisemblablement de ce schéma semi-tournant en volume large.

Certaines lames $(n=6)$ témoignent de l'intercalation d'enlèvements lamellaires en cours de production laminaire (fig. $6, \mathrm{n}^{\circ} 10$; fig. $10, \mathrm{n}^{\circ} 6$ ), alternance qui demeure toutefois épisodique. Par ailleurs, une continuité de la production laminaire à lamellaire est envisagée dans certains cas. Toutefois, les intentions de cette production lamellaire ne nous paraissent pas identiques à celle issue d'un débitage sur volume étroit. Les choix suivis dans la sélection des supports exploités (bloc, éclat ou plaquette) ne sont pas fixés par des facteurs économiques, mais pourraient s'inscrire dans des économies de production effectivement disjointes.

Dans la majeure partie des cas, les plans de frappe sont lisses et les corniches aménagées par de légères abrasions. En outre, le schéma général de production est générateur de nombreuses réfections de plan de frappe, ainsi qu'en témoigne l'importance numérique des tablettes de ravivage $(\mathrm{n}=75)$, présentes sous la forme de tablettes partielles $(n=67)$, de tablettes totales $(n=4)$ ou de fragments $(n=4)$ (fig. 7). Elles offrent un premier aperçu de la diversité et de l'évolution possible des lignes de front du débitage (fig. 8).

Les tablettes de ravivage indiquent une gestion semitournante des volumes débités. Certaines rendent compte de lignes de front du débitage très étroites, sur éclats ou plaquettes. L'extension de cette ligne de front au-delà du volume étroit (sur les flancs) semble, dans certains cas, liée à un contrôle du cintrage du nucléus, et non à un élargissement réel du volume exploité. Dans leur ensemble, l'étude des tablettes confirme cette dualité des schémas de production unipolaire avec, d'une part, l'exploitation de volumes fortement cintrés (éclats ou plaquettes) pour l'obtention de supports lamellaires et, d'autre part, l'exploitation de volumes plus ouverts, pour l'obtention de supports laminaires à lamellaires.
De plus rares produits viennent nuancer ce schéma général. Ceux-ci témoignent de la présence épisodique d'une gestion bipolaire de la production et, dans un cas, d'un agencement tournant du débitage lamellaire.

\section{TRANSFORMATION DES SUPPORTS}

D’un point de vue typologique, une certaine hiérarchisation des objectifs de la production se dégage. Les lamelles transformées par retouche unilatérale inverse (lamelles Dufour, sous-type Dufour) isolent ainsi un premier groupe typofonctionnel. Les supports lamellaires sélectionnés sont, dans ce cas de figure, des supports réguliers, rectilignes à peu courbes, issus d'un débitage centré ou, plus rarement, latéralisé (fig. 9). Les gabarits de lamelles $(\mathrm{n}=23)$ montrent des épaisseurs centrées autour de $2 \mathrm{~mm}$, des largeurs comprises entre $5 \mathrm{~mm}$ et $10 \mathrm{~mm}$ et des longueurs supérieures à 30/35 mm (maximum: $46 \mathrm{~mm}$ ). La latéralisation systématique du bord retouché (lamelles Dufour dextres) suggère une forte normalisation de leurs attributs fonctionnels.

La transformation de ces supports se fait par une retouche inverse très courte, continue ou partielle, généralement abrupte à semi-abrupte, plus rarement rasante. Elle tend à régulariser un bord à tendance rectiligne. Dans certains cas, cette retouche inverse dextre est associée à une autre directe senestre appointant la lamelle, souvent légèrement décentrée sur le bord «abattu» (bord gauche convergent, bord droit rectiligne). Cette régularisation de la morphologie des lamelles reste toutefois limitée et rappelle l'étroite imbrication des phases de production lamellaire et de réalisation des Dufour. Enfin, un petit nombre de lamelles $(\mathrm{n}=9)$, aux caractéristiques technomorphologiques voisines de celles transformées en Dufour, présente une retouche marginale du tranchant ou de la pointe. Ces corrections mineures de lamelles de plein débitage renvoient à la question de la fonction de l'outillage lamellaire (voir infra, p. 51).

Il se dégage des productions laminaires une diversité plus importante, pouvant suggérer une couverture plus large des besoins fonctionnels. Le corpus des lames présente un effectif composé d'environ $85 \%$ de produits d'une largeur comprise entre $11 \mathrm{~mm}$ et $17 \mathrm{~mm}$ et d'épaisseur comprise entre $2 \mathrm{~mm}$ et $5 \mathrm{~mm}$. Certaines grandes lames et fragments de lames larges peuvent toutefois être isolés. Ces lames présentent dans leur ensemble une allure très régulière, un profil rectiligne à légèrement courbe.

Ces lames sont dans leur ensemble peu transformées. Certains produits de plein débitage portent une encoche 

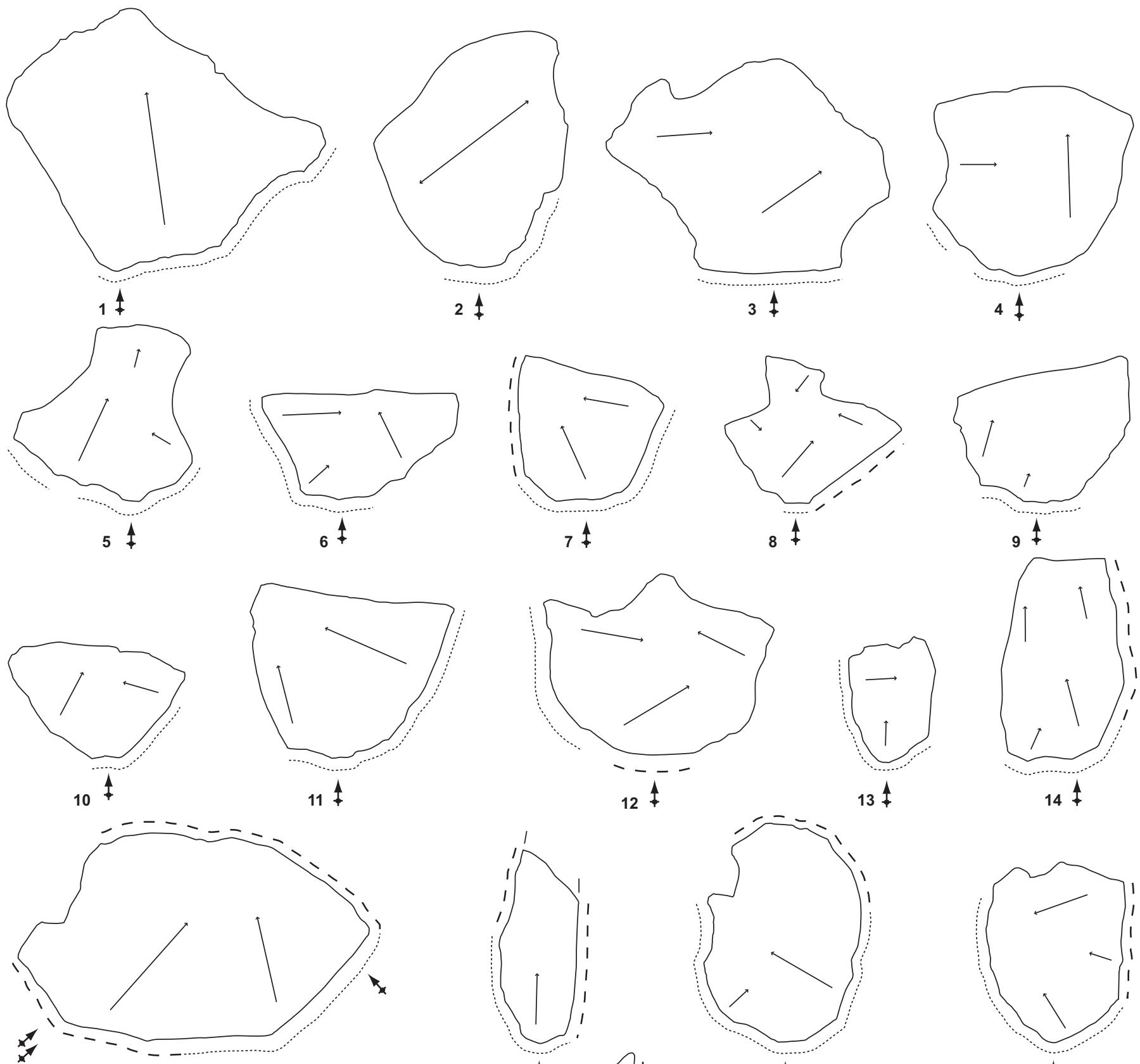

15
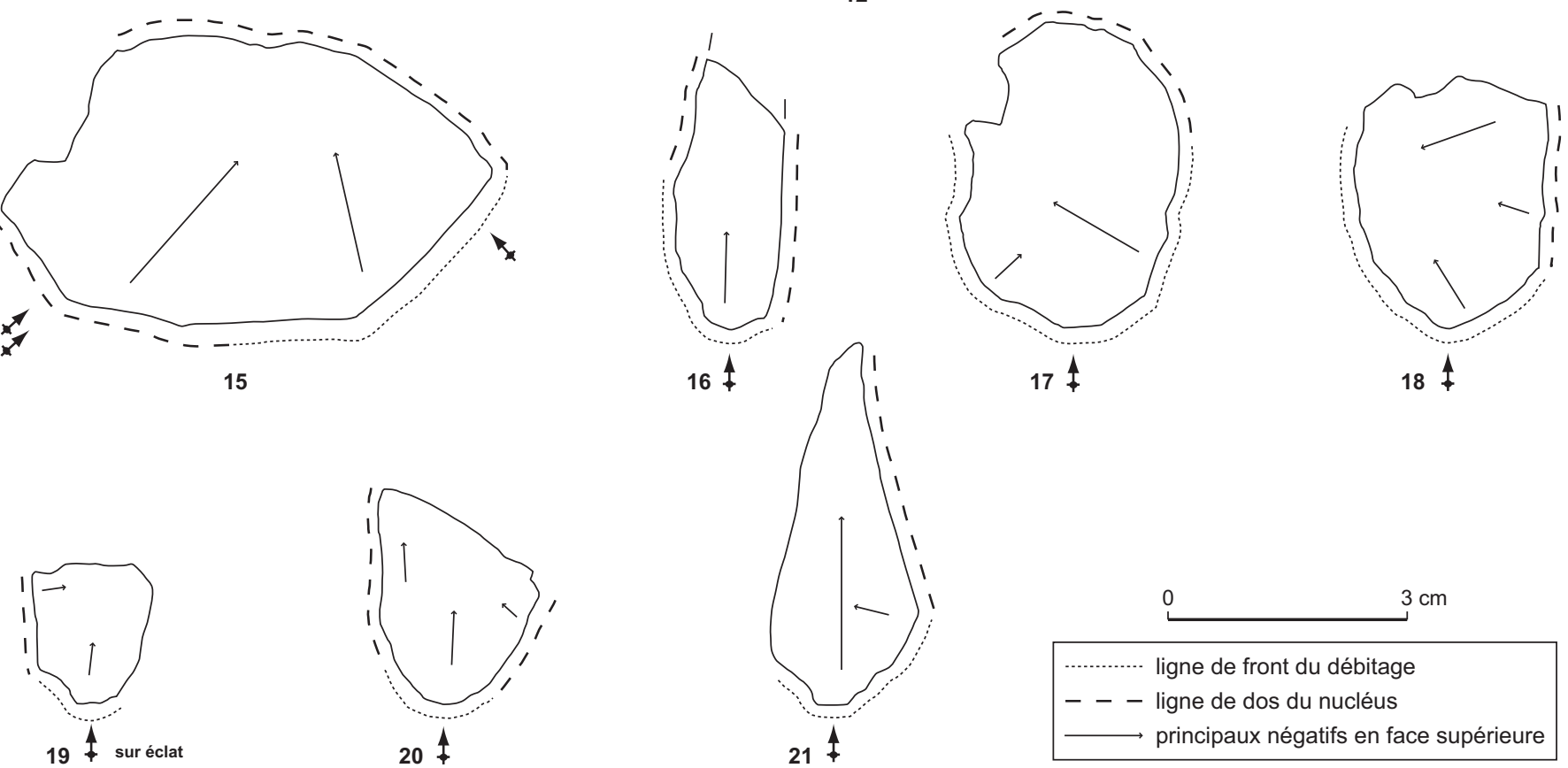

Fig. 8 - Front d'extension des débitages proto-aurignaciens «lamino-lamellaires» à la lecture des tablettes de ravivage $(1,8-14,16,17,20,21$ : foyer FG; 2, 3, 18 : foyer F; 4-7, 19: foyer $G ; 15$ : foyers FG et $G)$ (dessin: G. Porraz, Université de Tübingen). 

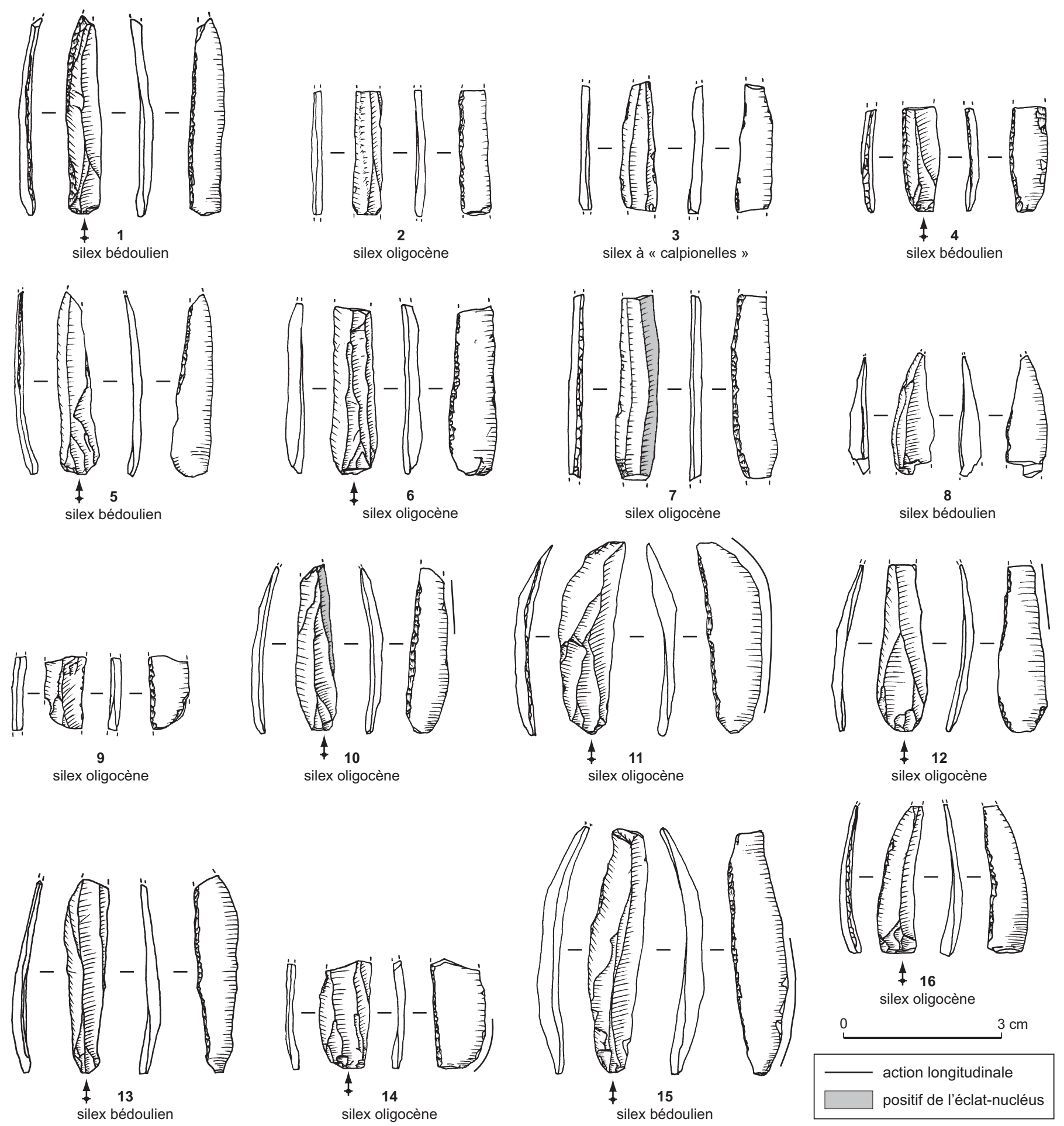

Fig. 9 - Lamelles Dufour du Proto-Aurignacien de la grotte de l'Observatoire: 1-9, population de lamelles type 1; 10-16, population de lamelles type 2; les fragments de lamelles 8 et 9 présentent une cassure d'impact caractéristique $(1-5,8-14$ : foyer FG; 6, 7, 16: foyer G; 15: foyer F) (dessin: G. Porraz, Université de Tübingen). 


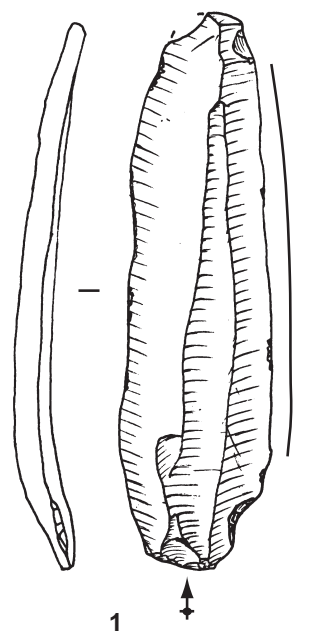

silex oligocène

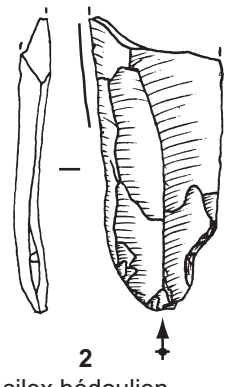

silex bédoulien

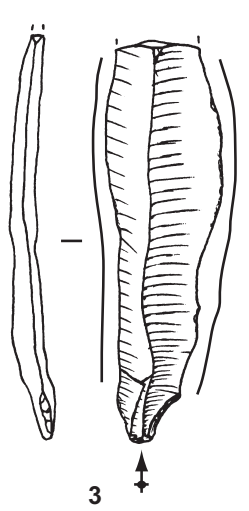

silex oligocène

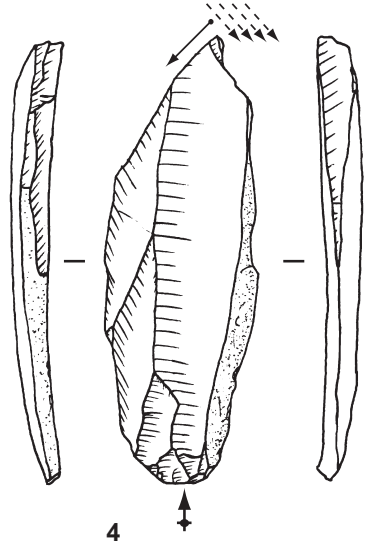

silex oligocène

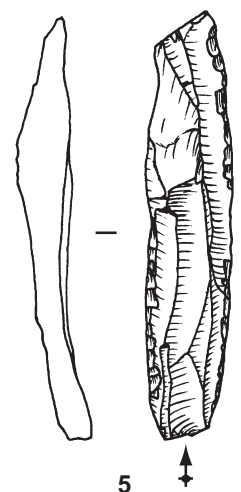

silex kimméridgien-portlandier

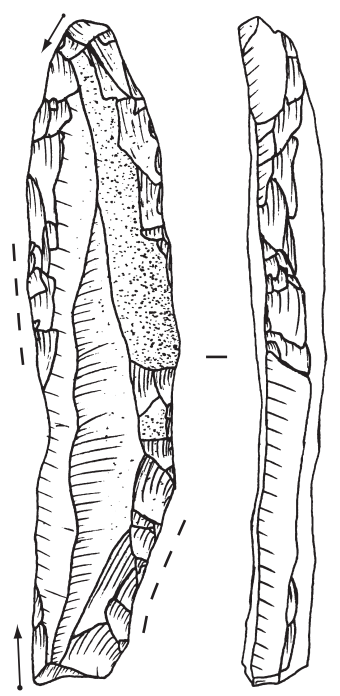

8

silex de la Scaglia Marchigiana

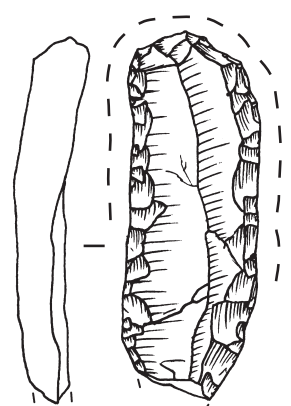

9

silex éocène

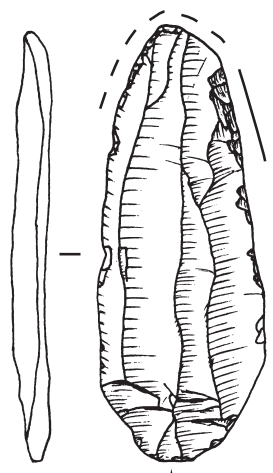

10

silex à « calpionelles »

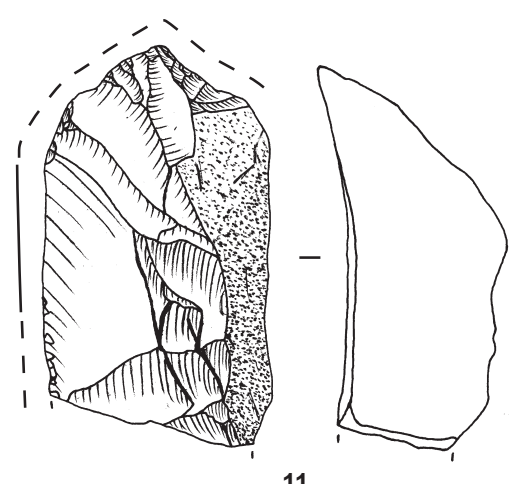

silex oligocène
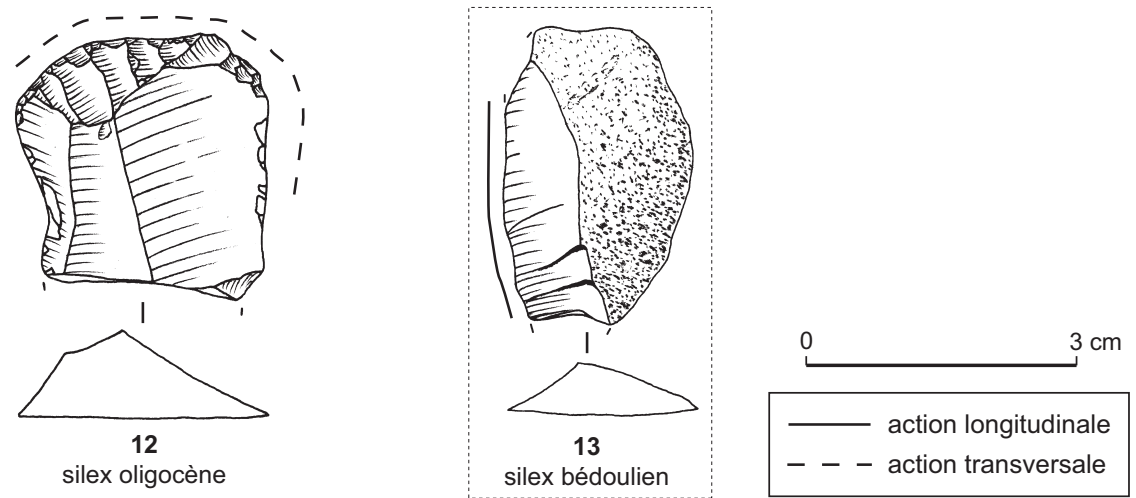

Fig. 10 - Produits retouchés du Proto-Aurignacien de la grotte de l'Observatoire: 1-3, lames à encoche retouchée; 4, burin dièdre; 5, lamelle à retouche bilatérale; 6, 7, 10, lames à retouche marginale; 8, lame intensément transformée; 9, grattoir sur lame retouchée; 11, grattoir caréné; 12, grattoir sur lame «épaisse»; 13, éclat cortical (1: foyer F; 2-12: foyer FG, 13 : foyer G) (dessin: G. Porraz, Université de Tübingen). 
retouchée généralement localisée à proximité immédiate du talon (fig. 10, n ${ }^{\text {os }} 1-3$ ), d'autres ont été transformées par une retouche peu envahissante et très localisée sur le tranchant (fig. 10, $\mathrm{n}^{\text {os }} 6,7$ et 10). Les lames à retouche épaisse sont plus rares: l'une d'entre elles (fig. 10, $\mathrm{n}^{\circ} 8$ ), reprises à ses deux extrémités par des enlèvements burinants, témoigne d'une exhaustion qui est à mettre en rapport avec l'éloignement du gîte de matière première (voir infra, p. 53). Les burins sont bien représentés, majoritairement sur cassure; deux grattoirs (un caréné, un en bout de lame épaisse) témoignent quant à eux d'un fond commun plus aurignacien (fig. $10, \mathrm{n}^{\text {os }} 11$ et 12 ).

\section{UTILISATION DES SUPPORTS : OBSERVATIONS PRÉLIMINAIRES}

Les résultats présentés ici constituent de premières pistes de lecture et d'interprétation fonctionnelle de l'outillage lamellaire, laminaire et sur éclat du Proto-Aurignacien de la grotte de l'Observatoire. Ces résultats préliminaires reposent sur l'analyse fonctionnelle de 217 pièces, dont 80 supports lamellaires (18 lamelles Dufour), 75 supports laminaires et 59 éclats. Cette étude s'inscrit dans le cadre d'une réflexion plus large portant sur la variabilité synchronique et diachronique de l'outillage lamellaire au début du Paléolithique supérieur (A. Pasquini, étude en cours).

L'effectif de lamelles Dufour étudiées peut être subdivisé en deux populations morphofonctionnelles distinctes:

- La première population étudiée $(n=7)$ regroupe des supports lamellaires allongés aux bords rectilignes (ou convergents), supports caractérisés par une largeur réduite et une allure élancée (fig. 9, $\mathrm{n}^{\text {os }} 1-9$ ). Au sein de ce groupe, des fractures et/ou des enlèvements typiques d'un impact lié à une utilisation en projectile (fig. $9, n^{\text {os }} 8$ et 9 ) ont été clairement identifiés $(\mathrm{n}=2)$.

- La seconde population étudiée $(n=11)$ se caractérise quant à elle par des supports lamellaires plus larges, courbes, au bord opposé à une retouche parfois convexe (fig. 9, $\left.\mathrm{n}^{\text {os }} 10-16\right)$. L'étude tracéologique indique l'emploi de ces lamelles en action de coupe, sur un matériau de dureté moyenne (fig. 9, $\mathrm{n}^{\text {os }} 10-12,14$ et 15 ).

Les lamelles non retouchées peuvent se répartir au sein de l'une ou l'autre de ces deux populations morphofonctionnelles. La présence de cassures d'impact a été reconnue sur des fragments de lamelles brutes de débitage $(n=2)$, conduisant ainsi à reconnaître une catégorie fonctionnelle (armature de projectile) au sein de laquelle l'élément typologique Dufour ne constituerait que l'élément le plus manifeste, et non l'unique expression. Dans cette même perspective, des supports lamellaires bruts aux bords réguliers présentent des traces d'action de coupe, plus rarement de raclage, de rainurage ou de perçage, individualisant une catégorie de lamelles à usage plus «domestique». La variabilité fonctionnelle ici introduite se fait l'écho de précédentes analyses (Broglio et al., 2005; O'Farrell, 2005; Garaizar, 2006).

Les états de conservation des supports laminaires ne permettent de proposer que des interprétations fonctionnelles limitées. La très nette prépondérance des actions de coupe au sein de cet ensemble doit notamment être interprétée à la lumière des filtres taphonomiques précédemment énoncés. Les lames les plus massives (utilisation sur une matière dure d'origine animale ou végétale) se distinguent toutefois des lames «légères », à l'extrémité aiguë, portant des traces de rainurage ou de perçage (fig. 6 et 10). Concernant le corpus typologique sur lame, il convient d'isoler l'ensemble "grattoir» qui présente des traces très développées liées au travail de matières dures d'origine animale (fig. 10, $\mathrm{n}^{\text {os }} 11,12$ ).

Le corpus «éclat», dont la production est pour l'essentiel dérivée des phases de mise en forme des productions laminaires et lamellaires (ne préjugeant en rien de leur production in situ), n'a fait l'objet d'aucune transformation par la retouche. Cependant leur insertion au sein du système fonctionnel est clairement attestée, avec près de $50 \%$ de l'effectif portant les traces d'une utilisation $(n=28 / 59)$. L'éclat cortical (fig. 10, n 13), associé à une activité de boucherie, est l'un de ces exemples.

\section{LES OCCUPATIONS}

PROTO-AURIGNACIENNES DE LA GROTTE DE L'OBSERVATOIRE DANS LE CONTEXTE MÉDITERRANÉEN : PREMIÈRE SYNTHÈSE

\section{UNE IDENTITÉ TEGHNIQUE PROTO-AURIGNACIENNE ?}

L'assemblage lithique des foyers $\mathrm{G}$ et $\mathrm{F}$ de la grotte de l'Observatoire se caractérise par les éléments techniques suivants :

- acquisition sélective de roches de bonne à très bonne qualité à la taille;

- coexistence de deux schémas de production: l'un laminaire, selon un schéma en volume large (production sur bloc ou plaquette); l'autre lamellaire, selon un schéma frontal/semi-tournant en volume étroit (production sur plaquette ou sur éclat); 
- unipolarité de la production; initialisation par réalisation d'une crête à un versant (schéma lamellaire) ; modalités d'entretien du cintre et de la carène assurées par le détachement des enlèvements eux-mêmes ainsi que par la réalisation de néo-crêtes (schéma laminaire); aménagement du point d'impact et entretien du plan de frappe selon une conception «lisse/abrasé»;

- relative monotonie du corpus typologique laminaire et lamellaire dominé par les lamelles Dufour, sous-type Dufour. Au sein de ces lamelles réalisées sur des supports allongés à tendance rectiligne, deux populations fonctionnelles peuvent être distinguées en fonction de leur étroitesse et de leur régularité;

- relative rareté $d u$ «fonds commun» aurignacien (grattoirs carénés, lames épaisses).

Les chaînes opératoires lithiques des foyers $\mathrm{G}$ et $\mathrm{F}$ de la grotte de l'Observatoire sont proches de celles aujourd'hui décrites dans la littérature pour les industries occidentales à grandes lamelles Dufour (Bazile, Sicard, 1999; Bon, Bodu, 2002; Bazile, 2005; Bordes, 2005; Ortega et al., 2005; Eizenberg, 2006; Slimak et al., 2006; Woerz, 2008). Ces caractéristiques communes appuient ainsi l'impression d'un complexe proto-aurignacien relativement homogène à l'échelle de l'Europe de l'Ouest (Bon, 2006; Slimak et al., 2006). Certains points de discussion méritent toutefois d'être introduits.

Si une continuité entre la production laminaire et lamellaire est soulignée dans le gisement de la grotte du Renne (Bon, Bodu, 2002), ces productions sont au contraire dissociées à la grotte Mandrin (Slimak et al., 2006) et à la grotte de l'Arbreda (Ortega et al., 2005). À la grotte de l'Observatoire, il semble bien exister une séparation entre les schémas laminaire et lamellaire. L'hypothèse d'une stricte dissociation des objectifs du débitage est toutefois partiellement discutée par la présence d'intercalations lamellaires en cours de productions laminaires, et par l'exploitation de plaquettes comprenant une production initiale de petites lames. Quoi qu'il en soit, l'opposition entre une production laminaire peu régulière et une production lamellaire très investie n'est pas vérifiée par les résultats de cette étude.

Les lamelles Dufour rectilignes et de grand module sont au cœur des objectifs du débitage lamellaire de la grotte de l'Observatoire. À cet égard, la relative monotonie du corpus typologique permet de distinguer la grotte de l'Observatoire de gisements marqués par une plus grande diversité du fonds typologique lamellaire (grotte de Fumane, abri du Piage couche K) (Broglio et al., 2005;
Bordes, 2005). Elle se distingue également des gisements de Mandrin et de l'Esquicho-Grapaou où une population de lamelles à dos de petit module a été reconnue (Bazile, Sicard, 1999; Slimak et al., 2006). L'existence de biais taphonomiques, écartée, ne peut être exclue, de même que l'hypothèse de biais d'ordre fonctionnel.

Si le Proto-Aurignacien d'Europe occidentale s'identifie comme un techno-complexe relativement homogène, l'étude des schémas de l'approvisionnement minéral révèle des comportements un peu plus contrastés. Toutefois, cette diversité apparaît subordonnée à la nature des espaces économiques occupés: approvisionnement local à voisin dans des contextes économiques favorables (grotte Mandrin, grotte de la Laouza, l'Esquicho-Grapaou), introduction de matières premières allochtones dans des contextes économiques peu favorables (grotte de l'Observatoire, grottes de l'Arbreda; voir Ortega, 2002). De façon paradoxale, ces réponses différentielles soulignent des choix technoéconomiques constants, basés sur une sélection quasi exclusive de matériaux de bonne à très bonne qualité à la taille. Dans des contextes peu favorables, l'exploitation de matières premières locales participerait alors d'un schéma économique de type «d'appoint» relevant de productions expédientes (productions d'éclats) (Ortega et al., 2005). Seules les occupations proto-aurignaciennes du gisement pyrénéen des Abeilles, avec une exploitation dominante de matières premières locales de qualité moyenne, rompraient partiellement ce schéma (Eizenberg, 2006).

\section{LES COMPORTEMENTS TEGHNIQUES DANS L'ESPACE}

La restitution des modalités de l'approvisionnement minéral à l'Observatoire repose sur la prise en compte de la totalité des vestiges lithiques abandonnés au cours de ses occupations, excluant tout biais d'origine spatiale. La fouille complète des niveaux proto-aurignaciens de la grotte de l'Observatoire confère donc une valeur absolue aux données de l'étude. Considérant la relative pauvreté numérique de l'industrie mise au jour (< 800 pièces), la première information retenue est celle d'un site protoaurignacien caractérisé par une faible densité de vestiges lithiques, suggérant des fréquentations épisodiques.

Les activités de taille identifiées à la grotte de l'Observatoire se limitent à de brèves séquences de production relevant principalement d'un schéma de débitage lamellaire. Les remontages effectués rendent compte d'une certaine diversité des opérations de taille. Celles-ci asso- 
cient des phases de mise en forme et d'entretien des nucléus (remontage d'éclats corticaux, présence et remontage de tablettes), des phases d'initialisation de la production (présence et remontage de produits à crête) et des phases de plein débitage (courte séquence de production). La diversité des opérations identifiées et des catégories technotypologiques représentées soulignent donc des modalités d'approvisionnement diversifiées : introduction de supports à débiter (éclats, plaquettes), de nucléus (à différents stades d'exploitation), de produits finis bruts ou retouchés (lames et lamelles).

Le nombre des tablettes de ravivage au sein de l'assemblage lithique de la grotte de l'Observatoire est particulièrement important $(n=75)$. Ces sous-produits techniques, qui constituent une catégorie techno-économique fixe, mettent inversement en lumière la mobilité et l'importance des nucléus au sein des stratégies d'approvisionnement des groupes proto-aurignaciens. Les décomptes et confrontations technologiques, les rapprochements et distinctions pétro-archéologiques soulignent des transports de matières premières impliquant d'une part le transit de nucléus par le site (nucléus «fantômes» lamellaires et, dans une moindre mesure, laminaires), d'autre part l'emport de certains produits issus de leurs phases d'exploitation.

Les caractéristiques de l'industrie lithique de la grotte de l'Observatoire donnent un aperçu tout à fait dynamique des modalités de l'approvisionnement minéral. Ces caractéristiques soulignent un fractionnement important des chaînes opératoires dans l'espace, fractionnement qui est à mettre en relation avec l'éloignement des gîtes de matières premières exploités (situés à plus de $130 \mathrm{~km}$ ). La diversité des produits technologiques impliqués dans le système fonctionnel (utilisation de lames, de lamelles, d'éclats mais aussi de produits corticaux, de lamelles à crête, de lames à néocrête, de produits "accidentés", fig. 6,9 et 10) témoigne d'un comportement économe vis-à-vis de matériaux introduits depuis des secteurs éloignés. Cette impression de diversité, issue de l'analyse technologique et tracéologique (voir supra, p. 39), ne plaide pas pour une spécialisation des registres d'activités dans le site.

La diversité pétrographique, associée à la forte estimation du NMB exploité ( $\mathrm{NMB}=59$ ), indique un assemblage lithique composé de multiples événements. L'ensemble des informations acquises (présence de divers carnivores, faible densité lithique, diversité des modalités d'approvisionnement, diversité pétrographique, brièveté des séquences de taille, absence de spécialisation claire des registres d'activité) indique des occupations répétées, plutôt de courte durée, par de petits groupes, et selon un schéma d'approvisionnement de type personnel (conservation des produits de site en site) (Kuhn, 1995). En ce sens, le profil de l'assemblage lithique témoigne de modalités d'occupation et d'approvisionnement renvoyant à un mode de subsistance basé sur une forte mobilité résidentielle et de longs déplacements.

\section{LES ESPACES ÉCONOMIQUES DE L'APPROVISIONNEMENT MINÉRAL}

$\mathrm{Au}$ vu des critères de sélection des matières premières, l'environnement proche de la grotte de l'Observatoire a représenté un espace économique peu favorable pour ces groupes proto-aurignaciens. Les matériaux locaux, représentés essentiellement par les galets siliceux du conglomérat de «I Ciotti», n'ont pas participé à l'approvisionnement minéral, y compris dans le cadre de productions «peu investies». Les occupations proto-aurignaciennes de la grotte de l'Observatoire se distinguent sur ce point de celles des sites voisins de Mochi et Bombrini (Negrino, Starnini 2003; Bietti, Negrino, 2007; Grimaldi, Porraz, étude en cours).

De cette étude se dégage assez clairement la possibilité de distinguer trois secteurs économiques (fig. 11). Ceux-ci témoignent d'une exploitation différentielle des espaces en fonction des besoins et moments de l'approvisionnement minéral. Cette partition géo-économique découle de l'organisation territoriale de ces populations, et la révèle.

Le premier secteur géographique est celui des silex de Provence occidentale, qui ont couvert plus de $85 \%$ des besoins de l'approvisionnement minéral. L'importance de ces matériaux, leur diversité de faciès, mais aussi la diversité technologique de leur forme d'introduction attestent une connaissance et une exploitation large, directe et/ou indirecte, des ressources lithiques de ce secteur. Compte tenu de l'importance de ces matériaux au sein des économies lithiques proto-aurignaciennes à l'Observatoire, la Provence occidentale peut être considérée comme le principal territoire occupé et exploité par ces populations.

Le second secteur géographique est celui des silex de Provence orientale, qui ont couvert moins de $10 \%$ des besoins de l'approvisionnement minéral. La quantité limitée de produits issus de ce secteur $(n=45)$ et la forte estimation du NMB $(\mathrm{NMB}=15)$ conduisent à privilégier l'hypothèse d'une introduction dominante de produits finis. Le spectre techno-économique des silex des formations du Kimméridgien-Portlandien, qui comportent un effectif important de nucléus lamellaires $(n=5 / 20)$ 


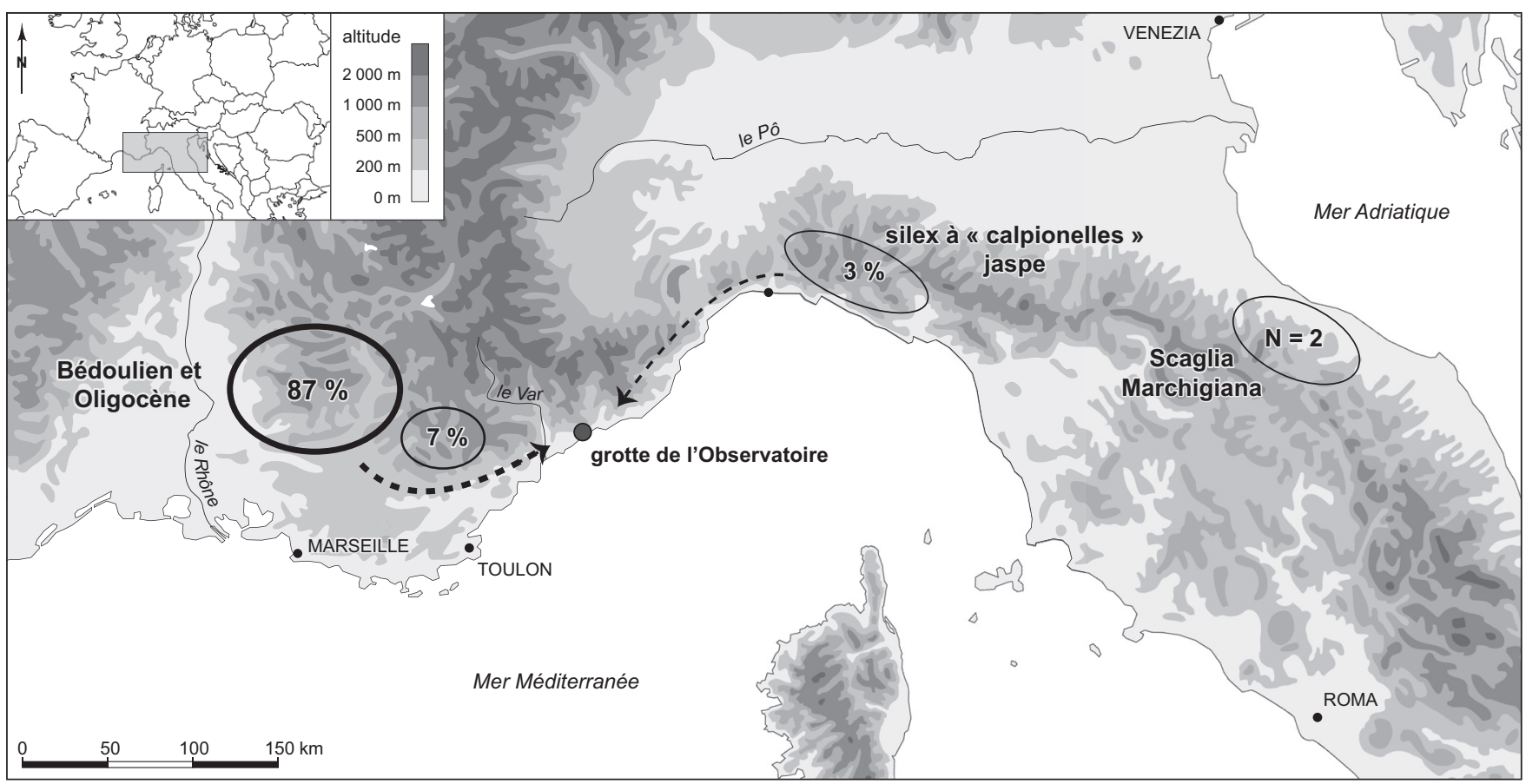

Fig. 11 - Localisation des secteurs géographiques de l'approvisionnement minéral dans le Proto-Aurignacien de la grotte de l'Observatoire (DAO: G. Porraz, Université de Tübingen).

(tabl. III), conduit à s'interroger sur la place effective de ces matériaux au sein des stratégies d'approvisionnement. Les évidences d'exploitation in situ de ces matériaux demeurent en effet relativement limitées. Une hypothèse serait d'accorder à ces matériaux le statut de produits reliquats, acquis lors de la fréquentation des secteurs préalpins périphériques, d'où ces silex sont originaires (milieu de moyenne montagne).

Le troisième secteur géographique est représenté par les roches siliceuses de Ligurie orientale et des Marches (moins de $5 \%$ de l'assemblage lithique). Exception faite des silex des formations «à calpionelles », les jaspes et les silex de la Scaglia Marchigiana sont représentés par des individus isolés, introduits sous forme de produits finis $(\mathrm{n}=6, \mathrm{NMB}=3)$. La pièce (fig. 10, $\mathrm{n}^{\circ} 8$ ), confectionnée sur un silex de la Scaglia Variegata Marchigiana (> $400 \mathrm{~km}$ ), témoigne d'un degré d'exhaustion inhabituel au sein de l'assemblage lithique. La présence de ces matériaux d'origine orientale peut être diversement interprétée. Soit celle-ci marque l'étendue des espaces économiques fréquentés par les groupes proto-aurignaciens, soit elle souligne l'existence de relations sociales étendues (contacts intergroupes), soit elle témoigne d'un moment différent dans l'histoire de l'occupation du site.

\section{LA GROTTE DE L'OBSERVATOIRE DANS SON CONTEXTE MÉDITERRANÉEN}

Les occupations proto-aurignaciennes constituent aujourd'hui les premières manifestations du Paléolithique supérieur dans l'arc liguro-provençal. Les observations archéologiques, du point de vue des systèmes de production, des économies de l'approvisionnement minéral ou encore des témoins symboliques, s'inscrivent assez manifestement en rupture avec les occupations moustériennes antérieures (Kuhn, Stiner, 1998; Onoratini, 2004; Bietti, Negrino, 2007; Porraz, Negrino, 2008). En outre, les niveaux d'occupation du Proto-Aurignacien sont séparés du Moustérien par la présence d'un plancher stalagmitique à l'Observatoire, et par la présence d'un horizon semi-stérile à Mochi et Bombrini. Avec les réserves nécessaires, ces éléments suggéreraient des discontinuités dans l'occupation humaine régionale et, consécutivement, une absence d'interactions culturelles dans cette zone spécifique.

Ce changement archéologique, dans l'arc liguroprovençal, marque le début du Paléolithique supérieur. Il s'inscrit dans le modèle global d'une origine allochtone du Proto-Aurignacien, qui serait alors associé à la première incursion des populations d'hommes anatomi- 
quement modernes sur le continent. Si cette hypothèse souffre de l'absence de résolutions chronologiques fines et d'associations anthropologiques indubitables, elle fait néanmoins valoir un certain nombre d'arguments scientifiques. Ceux-ci mettent en avant l'existence d'un fait technique commun au Proche- et Moyen-Orient et à l'Occident (la « lamelle à dos »), et une antériorité des industries orientales sur le Proto-Aurignacien, puis du Proto-Aurignacien sur l'Aurignacien ancien occidental (Bar-Yosef et al. 1996; Bon, 2002; Teyssandier, 2007).

La répartition géographique des sites proto-aurignaciens conduit alors à privilégier l'hypothèse d'une diffusion d'est en ouest le long de l'arc méditerranéen, diffusion volontiers considérée comme rapide (Mellars, 2005; BarYosef, 2006). En ce sens, la grotte de l'Observatoire peut contribuer à l'élaboration des scénarios et doit fournir des éléments régionaux de validation ou de réfutation.

Si le mécanisme de migration humaine participe de bien des hypothèses, les modèles et applications archéologiques à ce sujet n'ont été développés que par un nombre limité d'auteurs (Kelly, 1999 et 2003; Rockman, 2003; Davies, 2007). Outre la recherche des mécanismes en cause (pressions internes démographiques ou pressions externes du milieu), les études se sont consacrées à la formalisation des cadres archéothéoriques du processus de colonisation. Deux modèles sont généralement déclinés: l'un souligne une progression relativement continue par vagues («wave-ofadvance model») ; l'autre privilégie une avancée marquée par des incursions géographiques plus profondes («directional dispersal model»).

À l'instar des travaux menés sur les populations pionnières paléo-indiennes d'Amérique du Nord (Kelly, Todd, 1988), le scénario historique associé au Proto-Aurignacien devrait se confronter aux modèles et résultats issus des analyses techno-économiques. En ce sens, les données de la grotte de l'Observatoire se prêtent à un scénario de populations pionnières ayant circulé rapidement le long de l'arc méditerranéen. La faible densité de vestiges lithiques, le schéma d'une forte mobilité résidentielle et les longues circulations de matières premières, telles que perçues à la grotte de l'Observatoire, s'opposent à un modèle d'exploitation logistique reposant sur une forte implantation régionale.

Toutefois, ce champ d'hypothèses doit d'abord être réinséré dans le contexte géographique de l'étude. L'arc liguro-provençal, puisqu'il constitue une voie de circulation privilégiée (bande littorale) et qu'il présente des ressources minérales inégales, pourrait avoir induit des organisations territoriales elles aussi spécifiques (adaptation des organisations territoriales au milieu physique et économique). L'hypothèse d'une progression rapide du Proto-Aurignacien le long de l'espace méditerranéen, supportée par les schémas de mobilité et les distances cumulées de l'approvisionnement minéral, ne saurait donc a priori avoir de valeur extrarégionale.

Les données de la grotte de l'Observatoire trouvent cependant un certain écho dans la littérature. Les interprétations proposées dans les sites de Mandrin et de l'Arbreda, sur la base d'analyses techno-économiques, individualisent des occupations proto-aurignaciennes de courte durée. L'hypothèse d'un schéma de mobilité commun aux premières populations proto-aurignaciennes, impliquant une forte mobilité de petits groupes humains, pourrait être suggérée. Cette forte mobilité serait alors associée à de longs déplacements au sein de l'avant-pays méditerranéen, ainsi que le soulignent les données croisées de l'étude des grottes de l'Observatoire et de l'Arbreda au sein de laquelle $70 \%$ des matières premières ont une origine orientale (> $100 \mathrm{~km})$ (Ortega, 2002; Ortega et al., 2005).

Différents états et phases doivent être envisagés au sein d'un même processus. Les stratégies d'approvisionnement à la grotte de l'Observatoire reposent très majoritairement sur les silex du Bédoulien et de l'Oligocène de Provence occidentale. Cette économie des ressources minérales, à la grotte de l'Observatoire, atteste un ancrage des populations proto-aurignaciennes à l'ouest. Ce tropisme occidental est d'autant plus intéressant qu'il se comprend dans une dynamique de peuplement dont l'origine serait quant à elle orientale. Si les incertitudes liées aux cadres chronologiques effectivement considérés sont réelles, les implications portées par l'étude techno-économique de la grotte de l'Observatoire n'en sont pas moins intéressantes dans le cadre d'une approche du scénario de diffusion du Proto-Aurignacien. À un niveau régional en effet, ce tropisme occidental dans les schémas d'approvisionnement en matières premières lithiques à l'Observatoire impliquerait un franchissement relativement rapide de l'arc liguro-provençal par les populations proto-aurignaciennes, et un moment de stases en Provence occidentale (comprenant l'exploitation en retour de secteurs préalablement parcourus). Cette étude introduirait donc l'existence de discontinuités dans les rythmes de progression des populations d'hommes anatomiquement modernes en Europe de l'Ouest. Le prolongement de cette hypothèse serait donc l'existence d'une variabilité des schémas technoéconomiques proto-aurignaciens, variabilité régionale mais 
aussi diachronique (des phases) qui relèverait de ses dynamiques internes de diffusion.

\section{DISCUSSION}

- Le Proto-Aurignacien marque une rupture dans les occupations humaines de l'arc liguro-provençal, tant du point de vue des comportements de production que des modalités de l'approvisionnement minéral (Bietti, Negrino, 2007; Porraz, Negrino, 2008). À la grotte de l'Observatoire, ces modalités sont associées à l'exploitation sélective de gîtes de matières premières de très bonne qualité par de petits groupes circulant sur de longues distances. L'ensemble de ces observations va dans le sens de l'hypothèse de l'arrivée régionale d'une nouvelle population. Dans cette perspective, l'opportunité est donc offerte d'étudier les structures socio-économiques d'un changement technique qui ne serait ni le fruit d'une évolution locale, ni le résultat d'un transfert de connaissances.

- Une migration humaine implique l'existence d'organisations territoriales spécifiques, qu'elles relèvent d'une phase initiale d'occupation du territoire, ou d'une phase seconde de stabilisation (ou territorialisation) (Kelly, 2003; Davies, 2007). Au cours de ces phases, une variation ou une modification des expressions collectives, temporaire ou durable, doit être envisagée. À l'heure des synthèses, les éléments associés au Proto-Aurignacien d'Europe occidentale (faible densité de vestiges, occupations de courte durée, nature et importance des témoins d'expression symbolique, absence de restes humains) s'inscrivent dans la variabilité des schémas théoriques de la colonisation (voir Kelly, 1999 et 2003; Rockman, 2003; Davies, 2007). À l'inverse, l'Aurignacien ancien, qui lui succède en Europe occidentale, dévoile quant à lui la présence de nouvelles implantations, structures et expressions territoriales (Vanhaeren, d'Errico, 2006). La restitution des scénarios du changement implique donc une compréhension nouvelle des principes et expressions archéologiques des dynamiques de peuplement.

- La structure du Proto-Aurignacien s'articule autour de nouveaux comportements techniques et cynégétiques (Bon, 2005). Ce changement technique signale l'apparition de premières acquisitions sélectives, révélant une nouvelle organisation des comportements techniques dans l'espace. Ces changements, qui s'inscrivent dans la durée (Féblot-Augustins, 1997 et 2009), témoignent d'un rôle nouveau de la ressource lithique au sein des économies globales de subsistance. Un fait surprenant réside dans la présence d'une exploitation sélective des ressources dès ces premières phases du Paléolithique supérieur, impliquant la connaissance d'un territoire particulièrement étendu. Les comportements observés à la grotte de l'Observatoire appuient l'image d'un système rigide (exploitation exclusive de matériaux de très bonne qualité à la taille) dans le cadre d'organisations socio-économiques opérantes (les stratégies d'approvisionnement), mettant en jeu une exploitation des ressources sur un large territoire selon des modes d'acquisition directe et/ou indirecte.

- À l'échelle d'un continent et de plusieurs millénaires, les scénarios du changement déclinent des contextes physiques (voies de circulation, barrières physiques), économiques (répartition des ressources) et humains (présence/ absence, différences/similitudes) contrastés. La pluralité des scénarios révèle l'existence de plusieurs étapes, voire de plusieurs phases, au sein d'un même processus (Zilhao, d'Errico, 1999; Kozlowski, Otte, 2000; Conard, 2002; Bar-Yosef, 2006; Teyssandier et al., 2006; Tsanova, 2008). Ce processus historique est celui de la dispersion des populations d'hommes anatomiquement modernes. Au sein de cette thématique des dynamiques humaines de peuplement, l'arc euro-méditerranéen est une entité géographique incontournable. Les distances de transport des matières premières renseignées pour le Proto-Aurignacien (> $400 \mathrm{~km}$ ) n'ont à ce jour aucun équivalent extrarégional et confirment toute l'originalité et l'intérêt scientifique de ce secteur géographique (Porraz, sous presse). L'arc liguro-provençal est une porte physique vers l'Europe occidentale et constitue une alternative à la voie de diffusion danubienne septentrionale (Conard, Bolus, 2003). L'étude de son franchissement et la recherche des interactions culturelles et socio-économiques sont aujourd'hui indispensables pour une construction et une interprétation nouvelle des scénarios du peuplement à l'échelle européenne.

\section{Remerciements}

Cette étude a été financée par la région PACA et le conseil général des Alpes-Maritimes dans le cadre du Programme Collectif de Recherche «ETICALP» (Échanges, Transferts et Inter-Culturalités dans l'Arc Liguro-Provençal) placé sous la coordination de MM. D. Binder, R. Maggi et P. Simon. Cette étude a par ailleurs largement profité des compétences et infrastructures offertes par le laboratoire de l'UMR 6130 du CNRS (Centre d'études Préhistoire Antiquité Moyen Âge) à Valbonne. La rédaction de cet article s'est enrichie des commentaires et ajouts de deux relecteurs que nous remercions vivement. 


\section{BIBLIOGRAPHIE}

\section{BAR-YOSEF O}

2003: «Away from Home: Prehistoric Colonizations, Exchanges and Diffusions in the Mediterranean Basin", in VANDERMEERSCH B. (DIR.), Échanges et diffusion dans la Préhistoire méditerranéenne, Paris, éd. du Comité des travaux historiques et scientifiques, p. 71-82.

2006: «Neanderthals and Modern Humans: a Different Interpretation», in CONARD N. J. (DIR.), When Neanderthals and Modern Humans met, Tübingen, Kerns Verlag, p. 467-482.

BAR-YOSEF O., ARNOLD M., MERCIER N., BELFER-COHEN A., GOLDBERG P., Housley R., LAVille H., MEignen L., VOGEL J., VANDERMEERSCH B.

1996: «The Dating of the Upper Paleolithic Layers in Kebara Cave, Mount Carmel", Journal of Archaeological Science, 23, 2, p. 297-306

\section{BAZILE F.}

2005: «La composante lamellaire dans l'Aurignacien initial de la France méditerranéenne", in LE BRUN-RICALENS F. BORDES J. G., BON F. (DIR.), Productions lamellaires attribuées à l'Aurignacien: chaînes opératoires et perspectives technoculturelles, Actes du XIV congrès de l'UISPP, Liège 2-8 sept. 2001, Luxembourg, éd. du Musée national d'histoire et d'art (coll. ArchéoLogiques, 1), p. 325-336

\section{BAZILE F., SICARD S.}

1999: «Le premier Aurignacien du Languedoc oriental dans son contexte méditerranéen", in SACCHI D. (DIR.), Les Faciès leptolithiques du nord-ouest méditerranéen: milieux naturels et culturels, Actes du XXIV congrès préhistorique de France, Carcassonne 26-30 sept. 1994, Paris, Société préhistorique française, p. 117-125.

\section{BietTi A., NEgRino F.}

2007: «Transitional Industries from Neandertals to Anatomically Modern Humans in Continental Italy: Present State of Knowledge ", in RIEL-SALVATORE J., ClARK G. A. (DIR.), New approaches to the study of Early Upper Palaeolithic "transitional" industries in Western Eurasia, Oxford, Archaeopress (coll. British Archaeological Reports, International Series, 1620), p. 41-60.
BINDER D. avec la collab. de BARBIER M., GUILBERT R.

1994: Recensement des disponibilités en matières premières lithiques dans la région PACA, rapport de prospection thématique, conservé à la médiathèque de l'architecture et du patrimoine de Saint-Cyr.

1998: Recensement des disponibilités en matières premières lithiques dans la région ProvenceAlpes-Côte d'Azur, rapport de synthèse sur les prospections thématiques en Provence rhodanienne 1995-1997, Service régional de l'archéologie Provence-Alpes-Côte d'Azur.

BON F.

2002: L'Aurignacien entre mer et océan: réflexion sur l'unité des phases anciennes de l'Aurignacien dans le sud de la France, Paris, Société préhistorique française (coll. Mémoires de la Société préhistorique française, 29), $253 \mathrm{p}$.

2005: «Little Big Tool: enquête autour du succès de la lamelle», in LE BRUNRICALENS F., BORDES J.-G., BON F. (DIR.) Productions lamellaires attribuées à l'Aurignacien: chaînes opératoires et perspectives technoculturelles, Actes du XIV congrès de l'UISPP, Liège 2-8 sept. 2001, Luxembourg, éd. du Musée national d'histoire et d'art (coll. ArchéoLogiques, 1), p. 479-484

2006: «A Brief Overview of Aurignacian Cultures in the Context of the Industries of the Transition from the Middle to the Upper Paleolithic », in BAR-YOSEF O., ZILHAO J. (DIR.), Towards a Definition of the Aurignacian, Proceedings of the Symposium held in Lisbon, Portugal, June 25-30, 2002, Lisbonne, American School of Prehistoric Research/Instituto Português de Arqueologia (coll. Trabalhos de Arqueologia, 45), p. 133-144.

\section{BON F., BODU P.}

2002: "Analyse technologique du débitage aurignacien", in SCHMIDER B. (DIR.) L'Aurignacien de la grotte du Renne: les fouilles d'André Leroi-Gourhan à Arcy-surCure (Yonne), Paris, CNRS Éditions (coll. supplément à Gallia Préhistoire, XXXIV), p. 115-133.

\section{BORDES J.-G.}

2005: «La séquence aurignacienne du nord de l'Aquitaine: variabilité des productions lamellaires à Caminade-est, Roc-deCombe, le Piage et Corbiac-Vignoble II ", in LE BRUN-RICALENS F., BORDES J.-G.,
BON F. (DIR.), Productions lamellaires attribuées à l'Aurignacien: chaînes opératoires et perspectives technoculturelles, Actes du XIV congrès de l'UISPP, Liège 2-8 sept. 2001, Luxembourg, éd. du Musée national d'histoire et d'art (coll. ArchéoLogiques, 1), p. 123-154

BORDES J.-G., LE BRUN-RICALENS F., Castel J.-C., DUCasse S., Faivre J.-P., FERUGLIO V., HENRY-GAMBIER D.,

LACRAMPE-CUYAUBÈRE F.,

LAROULANDIE V., LENOBLE A., martin H., Maureille B., Morala A. MORIN E., RENARD C., RENDU W., RIGAUD S., ROUGIER H., SZMIDT C., TARTAR E., TEXier J.-P., TEYSSANDIER N.

2008: "Les débuts du Paléolithique supérieur dans le sud-ouest de la France: fouilles 2004-2006 au Piage (Fajoles, Lot). Problématique et premiers résultats", in JAUBERT J., BORDES J.-G., ORTEGA I. (DIR.), Les Sociétés du Paléolithique dans un grand sud-ouest de la France: nouveaux gisements, nouveaux résultats, nouvelles méthodes, Paris, Société préhistorique française (coll. Mémoire de la Société préhistorique française, XLVII), p. 261-288.

Boule M., VilleneUVe L. DE

1927: La Grotte de l'Observatoire à Monaco, Monaco, Institut de paléontologie humaine/Masson (coll. Archives de l'Institut de paléontologie humaine, Mémoires 39), 113 p.

Broglio A., Bertola S., De Stefani M., MARINI D., LEMORINI C., ROSSETTI P.

2005: «La production lamellaire et les armatures lamellaires de l'Aurignacien ancien de la grotte de Fumane (monts Lessini, Vénétie)", in LE BRUN-RICALENS F., BORDES J.-G., BON F. (DIR.), Productions lamellaires attribuées à l'Aurignacien: chaînes opératoires et perspectives technoculturelles, Actes du XIV congrès de l'UISPP, Liège 2-8 sept. 2001, Luxembourg, éd. du Musée national d'histoire et d'art (coll. ArchéoLogiques, 1), p. 415-436.

Cancellieri e., Peresani M., Poggi G., Silvestrini M., LUCiani V., SCOTTI M.

À paraître: Distribution, Suitability and Characterization of Flint Sources in the Marche Appenine (Italy): the Upper Esino Basin, Hugo Obermaier Society for Quaternary Research and Archaeology of the Stone Age, 51 st annual meeting 
in Ljubljana (Slovenia), 14 th-18 th April 2009, Poster Session.

\section{CONARD N. J.}

2002: «The Timing of Cultural Innovations and the Dispersal of Modern Humans in Europe», Terra Nostra, 6, p. 82-94.

\section{CONARD N. J., BOLUS M.}

2003: «Radiocarbon Dating the Appearance of Modern Humans and Timing of Cultural Innovations in Europe: New Results and New Challenges", Journal of Human Evolution, 44, p. 331-371.

\section{DAVIES W.}

2007: «Re-evaluating the Aurignacian as an Expression of Modern Human Mobility and Dispersal", in MELLARS P., Boyle K., Bar-Yosef O., STRINGer C. (DIR.), Rethinking the Human Revolution: New Behavioural and Biological Perspectives on the Origin and Dispersal of Modern Humans, Cambridge, McDonald Institute for Archaeological Research, p. 263-274.

\section{DEL SOLDATO M.}

1990: "Analisi petrographiche dell'industria litica scheggiata», in MAGGI R. (DIR.), Archeologia dell'Appennino Ligure, Bordighera, Istituto Internazionale di Studi Liguri, p. 207-218.

Del Soldato M., Garibaldi P., Maggi R. 1987: «Ricerche petrografiche», in MELLI P., DEL LUCCHESE A. (DIR.), Archeologia in Liguria III.1 Scavi e scoperte 19821986: Preistoria e protostoria, Genova, Soprintendenza archeologica della Liguria, p. 181-184.

\section{EIZENBERG L.}

2006: Le Rôle de l'Aurignacien archaïque dans les phases initiales du Paléolithique supérieur: apport des industries lithiques de la grotte des Abeilles (Haute-Garonne), Mémoire de Master II, Université de Toulouse Le Mirail.

\section{FÉBLOT-AUguSTINS J.}

1997: La Circulation des matières premières au Paléolithique, Liège, ERAUL, 2 vol., 275 p. 2009: «Revisiting European upper Paleolithic Raw Material Transfers: the Demise of the Cultural Ecological Paradigm?" in ADAMS B., BlAdES B. S. (DIR.), Lithic Materials and Paleolithic Societies, WileyBlackwell, p. 25-46.

\section{GARAIZAR J. R.}

2006: Industria litica y sociedad en la transicion del Paleolitico Medio al Superior en torno al Golfo de Bizkaia, Thèse de Doctorat, Universidad de Cantabria, Departamento de Ciencias Historicas, Faculdad de Filosofia y Letras, $561 \mathrm{p}$.

\section{GUILLOMET-MALMASSARI V.}

2009: Continuités, discontinuités: l'approche des transitions en Préhistoire, Thèse de Doctorat, Université de Nanterre Paris-X, $216 \mathrm{p}$.

\section{KELLY R. L.}

1999: «Hunter-gatherer Foraging and Colonization of the Western Hemisphere», Anthropologie, 37, 2, p. 143-153.

2003: "Colonization of New Land by Hunter-gatherers. Expectations and Implications Based on Ethnographic Data », in ROCKMAN M., STEELE J. (DIR.), Colonization of Unfamiliar Landscapes: The Archaeology of Adaptation, London and New York, Routledge, p. 44-58.

KELLY R. L., TODD L. C.

1988: «Coming into the Country: Early Paleo-Indian Hunting and Mobility», American Antiquity, 53, 2, p. 231-244.

\section{KOZLOWSKI J. K., OTTE M.}

2000: «The Formation of the Aurignacian in Europe", Journal of Anthropological Research, 56, p. 513-534.

KUHN S. L.

1995: Mousterian Lithic Technology: An Ecological Perspective, Princeton, Princeton University Press, 209 p.

KUHN S. L., STINER M. C.

1998: "The earliest Aurignacian of Riparo Mochi (Liguria, Italy) », Current Anthropology, 29, 3, p. 175-189.

\section{LAPLACE G.}

1966: Recherche sur l'origine et l'évolution des complexes leptolithiques, Rome, École française de Rome (coll. Mélanges d'archéologie et d'histoire, Suppl. 4), p. 586.

\section{Mellars P.}

2004: «Neanderthals and the Modern Human Colonization of Europe », Nature, 432, p. 461-465.

2005: "The Impossible Coincidence: a Single-Species Model for the Origins of Modern Human Behavior in Europe», Evolutionary Anthropology, 14, p. 12-27.

2006: "Going East: New Genetic and Archaeological Perspectives on the Modern Human Colonization of Eurasia», Science, 313, p. 796-800.

\section{NEGRINO F.}

2006: «Riparo Bombrini, Balzi Rossi (Ventimiglia, Imperia): la campagna 2005 », Ligures, 3, p. 194-196.

\section{NEGRINo F., STARNini E.}

2003: "Patterns of Lithic Raw Material Exploitation in Liguria from the Palaeolithic to the Copper Age», Préhistoire du Sud-Ouest, 5, p. 235-243.

\section{O'FARRELl M.}

2005: «Étude préliminaire des éléments d'armature lithique de l'Aurignacien ancien de Brassempouy", in LE BRUNRICALENS F., BORDES J.-G., BON F. (DIR.), Productions lamellaires attribuées à l'Aurignacien: chaînes opératoires et perspectives technoculturelles, Actes du XIV congrès de l'UISPP, Liège 2-8 sept. 2001, Luxembourg, éd. du Musée national d'histoire et d'art (coll. ArchéoLogiques, 1), p. 395-412.

\section{ONORATINI G.}

2004: «Le Proto-Aurignacien: première culture de l'homme moderne de Provence et Ligurie", L'Anthropologie, 108, p. 239-249.

\section{ONORATINI G., SIMON P.}

2006: «Le Proto-Aurignacien de la grotte de l'Observatoire (principauté de Monaco) ", in SANCHIDRIAN J.L., MARQUeZ A.-M., FILlOLA J.-M. (DIR.), La Cuenca mediterranea durante el Paleolitico superior, Reunion de la VII Comission Paleolitico superiore, IV Simposio Cueva de Nerja, UISPP, p. $430-444$.

\section{ONORATINI G., Simon P., SimONe S.}

1999: «Mise en évidence du ProtoAurignacien à la grotte de l'Observatoire", Bulletin du musée d'Anthropologie préhistorique de Monaco, 40, p. 43-56.

\section{ORTEGA D.}

2002: «Mobilitat i desplaçaments dels grups caçadors-recollectors a inicis del Paleolitic superior a la regio Pirinenca oriental», Cypsela, 14, p. 11-26.

OrTega Cobos D., SOler MASFERrer N., MAROTO GENOVER J.

2005: «La production des lamelles pendant l'Aurignacien archaïque dans la grotte de l'Arbreda: organisation de la production, variabilité des méthodes et des objectifs ", in LE BRUN-RICALENS F., BORDES J.-G., BON F. (DIR.), Productions lamellaires attribuées à l'Aurignacien: chaînes opératoires et perspectives technoculturelles, Actes $d u$ XIVE congrès de l'UISPP, Liège 2-8 sept. 2001, 
Luxembourg, éd. du Musée national d'histoire et d'art (coll. ArchéoLogiques, 1), p. 359-373.

\section{PORRAZ G.}

2005: En marge du milieu alpin: dynamiques de formation des ensembles lithiques et modes d'occupation des territoires au Paléolithique moyen, Thèse de Doctorat, Université de Provence, $386 \mathrm{p}$.

À paraître: «Regards croisés: la diffusion des jaspes de Ligurie orientale (Italie) et l'approvisionnement en matières premières lithiques à l'abri Pié Lombard (Paléolithique moyen, France)", in Conard N. J., Delagnes A. (DIR.), Settlement Dynamics IV, Tübingen, Kerns Verlag.

\section{Porraz G., Negrino F.}

2008: «Espaces économiques et approvisionnement minéral au Paléolithique moyen dans l'aire liguro-provençale », in Binder D., Delestre X., PERGOla P. (DIR.), Archéologies transfrontalières (Alpes du Sud, Côte d'Azur, Piémont et Ligurie): bilan et perspectives de recherche, Actes du colloque, Nice, 13-15 déc. 2007, Monaco, Musée d'anthropologie de Monaco, p. $29-40$.

\section{PORRAZ G., Simon P., TOMASSO A.}

2009: "Bilan documentaire sur les formations à silex du KimméridgienPortlandien de Provence orientale (Var et Alpes-Maritimes)", in BINDER D., MAGgI R., SIMON P., Le PCR ETICALP: échanges, transferts, inter-culturalités dans l'arc liguro-provençal, Rapport d'acti- vité, Service régional de l'archéologie Provence-Alpes-Côte d'Azur.

\section{ROCKMAN M.}

2003: "Knowledge and Learning in the Archaeology of Colonization", in ROckman M., STEEle J. (DIR.), Colonization of Unfamiliar Landscapes: the Archaeology of Adaptation, London and New York, Routledge, p. 3-24.

\section{Slimak L., Pesesse D., Giraud Y.}

2006: «La grotte Mandrin et les premières occupations du Paléolithique supérieur en Occitanie orientale", in BON F., MAILlO FERnANDEZ J. M., ORTEGA Cobos D. (DIR.), Autour des concepts de Proto-Aurignacien, d'Aurignacien initial et ancien: unité et variabilité des comportements techniques des premiers groupes d'hommes modernes dans le sud de la France et le nord de l'Espagne, Actes de la table ronde, Toulouse 2003, Madrid, Espacio, tiempo y forma, p. $237-259$.

\section{TEYSSANDIER N.}

2007: «L'émergence du Paléolithique supérieur en Europe: mutations culturelles et rythmes d'évolution ", Paléo, 19, p. 367-389.

Teyssandier N., Bolus M., Conard N. J. 2006: «The Early Aurignacian in Central Europe and its Place in a European Perspective ", in BAR-YOSEF O., ZILHAO J. (DIR.), Towards a Definition of the Aurignacian, Proceedings of the Symposium held in Lisbon, Portugal, June 25-30, 2002, Lisbonne, American School of
Prehistoric Research/Instituto Português de Arqueologia (coll. Trabalhos de Arqueologia, 45), p. 9-29.

\section{TSANOVA T.}

2008: Les Débuts du Paléolithique supérieur dans l'est des Balkans: réflexion à partir de l'étude taphonomique et techno-économique des ensembles lithiques des sites de Bacho Kiro (couche 11), Temnata (couches VI et 4) et Kozarnika (niveau VII), Oxford, Archaeopress (coll. BAR International Series, 1752), $325 \mathrm{p}$.

\section{VANHAEREN M., D'ERRICO F.}

2006: «Aurignacian Ethno-linguistic Geography of Europe Revealed by Personal Ornaments", Journal of Archaeological Science, 33, 8, p. 1105-1128.

\section{WOERZ P.}

2008: «A Reassessment of the Aurignacian of the grotte du Figuier (SaintMartin-d'Ardèche, Ardèche, France) ", Archäologisches Korrespondenzblatt, 38, p. 301-317.

\section{ZILHAO J.}

2006: "Neandertals and Modern Mixed, and It Matters», Evolutionary Anthropology, 15, p. 183-195.

\section{ZILHAO J., D'ERRICO F.}

1999: "The Chronology and Taphonomy of the Earliest Aurignacian and its Implications for the Understanding of Neandertal Extinction ", Journal of World Prehistory, 13, 1, p. 1-68. 